\title{
HOMOLOGY PRO STABILITY FOR TOR-UNITAL PRO RINGS
}

\author{
RYOMEI IWASA
}

\begin{abstract}
Aвstract. Let $\left\{A_{m}\right\}$ be a pro system of associative commutative, not necessarily unital, rings. Assume that the pro systems $\left\{\operatorname{Tor}_{i}^{\mathbb{Z} \ltimes A_{m}}(\mathbb{Z}, \mathbb{Z})\right\}_{m}$ vanish for all $i>0$. Then we prove that the sequence $\left\{H_{l}\left(\mathrm{GL}_{n}\left(A_{m}\right)\right)\right\}_{m} \rightarrow\left\{H_{l}\left(\mathrm{GL}_{n+1}\left(A_{m}\right)\right)\right\}_{m} \rightarrow\left\{H_{l}\left(\mathrm{GL}_{n+2}\left(A_{m}\right)\right)\right\}_{m} \rightarrow \cdots$ stabilizes up to pro isomorphisms for $n$ large enough than $l$ and the stable range of $A_{m}$ 's.
\end{abstract}

\section{Contents}

0. Introduction 1

1. Pro stability for $K_{1} \quad 4$

2. Tor-unital pro rings $\quad 7$

3. Pro acyclicity of triangular spaces 9

4. Homology pro stability 18

References $\quad 28$

\section{InTRODUCTION}

0.1. The homology stability for general linear groups is a simple but deep question on homological algebra. Let $R$ be an associative unital ring. We consider the general linear groups $\operatorname{GL}_{n}(R)$ of $R$ and their sequence

$$
\mathrm{GL}_{n}(R) \hookrightarrow \mathrm{GL}_{n+1}(R) \hookrightarrow \mathrm{GL}_{n+2}(R) \hookrightarrow \cdots,
$$

where each embedding is given by sending $\alpha$ to $\left(\begin{array}{cc}\alpha & 0 \\ 0 & 1\end{array}\right)$. The question is whether the induced sequence of the integral group homology

$$
H_{l}\left(\mathrm{GL}_{n}(R)\right) \rightarrow H_{l}\left(\mathrm{GL}_{n+1}(R)\right) \rightarrow H_{l}\left(\mathrm{GL}_{n+2}(R)\right) \rightarrow \cdots
$$

stabilizes for $n$ large enough than $l$. There have been many works on this problem, and the most striking result was obtained by Suslin.

Theorem 0.1 (Suslin [Su82]). Let $R$ be an associative unital ring and $l \geq 0$. Then the canonical map

$$
H_{l}\left(\mathrm{GL}_{n}(R)\right) \rightarrow H_{l}\left(\mathrm{GL}_{n+1}(R)\right)
$$

is surjective for $n \geq \max (2 l, l+\operatorname{sr}(R)-1)$ and bijective for $n \geq \max (2 l+1, l+\operatorname{sr}(R))$, where $\operatorname{sr}(R)$ is the stable range of $R$.

Things become much harder and interesting if we consider non-unital rings. Then the homology stability is strongly related with the $K$-theory excision and the Tor-unitality.

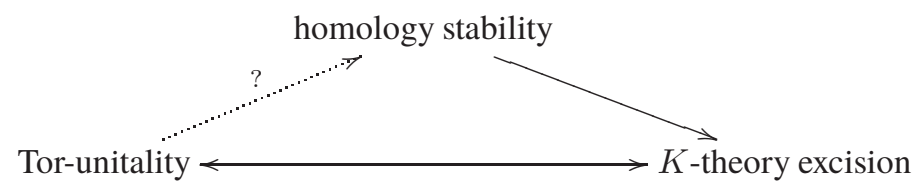

Let $R$ be an associative unital ring and $A$ a two-sided ideal of $R$. We define the $n$-th relative $K$-group by

$$
K_{n}(R, A):=\pi_{n} \operatorname{hofib}\left(B \mathrm{GL}(R)^{+} \rightarrow B \mathrm{GL}(R / A)^{+}\right) .
$$


We say that $A$ satisfies the $K$-theory excision if, for every unital ring $R$ which contains $A$ as a two-sided ideal and for every $n \geq 1$, the canonical map

$$
K_{n}(\mathbb{Z} \ltimes A, A) \stackrel{\sim}{\rightarrow} K_{n}(R, A)
$$

is an isomorphism. It is well-known that the $K$-theory excision fails in general. However, if the homology $H_{l}\left(\mathrm{GL}_{n}(A)\right)$ stabilizes for $n$ large enough, then $A$ satisfies the $K$-theory excision. Such being the case, the homology stability for non-unital rings fails in general, even if the stable range of $A$ is finite.

On the other hand, in [Su95], Suslin has completely determined the obstruction to the $K$-theory excision: An associative ring $A$ satisfies the $K$-theory excision if and only if $A$ is Tor-unital, i.e. $\operatorname{Tor}_{i}^{\mathbb{Z} \ltimes A}(\mathbb{Z}, \mathbb{Z})=$ 0 for all $i>0$. Hence, we may hope that Tor-unital rings satisfy the homology stability. Again, Suslin has given a partial solution.

Theorem 0.2 (Suslin $[$ Su96]). Let $A$ be a Tor-unital $\mathbb{Q}$-algebra, $r=\max (\operatorname{sr}(A), 2)$ and $l \geq 0$. Then the canonical map

$$
H_{l}\left(\mathrm{GL}_{n}(A)\right) \rightarrow H_{l}\left(\mathrm{GL}_{n+1}(A)\right)
$$

is surjective for $n \geq 2 l+r-2$ and bijective for $n \geq 2 l+r-1$.

Unfortunately, commutative rings really happen to be Tor-unital. Instead, a recent trend has been to think Tor-unital pro rings. We say that a pro system $\left\{A_{m}\right\}$ of associative rings is Tor-unital if the pro system $\left\{\operatorname{Tor}_{i}^{\mathbb{Z} \ltimes A_{m}}(\mathbb{Z}, \mathbb{Z})\right\}_{m}$ vanish for all $i>0$. A notable discovery by Morrow [Mo15] is that, for any ideal $A$ of a noetherian commutative ring, the pro ring $\left\{A^{m}\right\}_{m \geq 1}$ is Tor-unital. Besides, GeisserHesselholt [GH06] has generalized Suslin's excision theorem to the pro setting: If $\left\{A_{m}\right\}$ is a Tor-unital pro ring then the canonical map

$$
\left\{K_{n}\left(\mathbb{Z} \ltimes A_{m}, A_{m}\right)\right\}_{m} \stackrel{\sim}{\rightarrow}\left\{K_{n}\left(R_{m}, A_{m}\right)\right\}_{m}
$$

is a pro isomorphism for any pro system of unital rings $\left\{R_{m}\right\}$ with a level map $\left\{A_{m}\right\} \rightarrow\left\{R_{m}\right\}$ which exhibits each $A_{m}$ as a two-sided ideal of $R_{m}$.

Our main theorem is a pro version of Theorem 0.2 .

Theorem 0.3. Let $\left\{A_{m}\right\}$ be a commutative Tor-unital pro ring ${ }^{1}, r=\max \left(\operatorname{sr}\left(A_{m}\right), 2\right)$ and $l \geq 0$. Then the canonical map

$$
\left\{H_{l}\left(\mathrm{GL}_{n}\left(A_{m}\right)\right)\right\}_{m} \rightarrow\left\{H_{l}\left(\mathrm{GL}_{n+1}\left(A_{m}\right)\right)\right\}_{m}
$$

is a pro epimorphism for $n \geq 2 l+r-2$ and a pro isomorphism for $n \geq 2 l+r-1$.

It follows from Theorem 0.3 that if $\left\{A_{m}\right\}$ is commutative Tor-unital then the action of $\mathrm{GL}_{n}(\mathbb{Z})$ on $\left\{H_{l}\left(\mathrm{GL}_{n}\left(A_{m}\right)\right)\right\}_{m}$ is pro trivial for $n \geq 2 l+r-1$, cf. Corollary 4.13. Together with the standard argument this reproves Geisser-Hesselholt's pro excision theorem for commutative Tor-unital rings of finite stable range.

0.2. Outline. Suslin used the Malcev theory in the proof of Theorem 0.2 , which works only for $\mathbb{Q}$ algebras. More precisely, he used the Malcev theory to get an acyclicity of the union of triangular spaces, cf. [Su96, Corollary 5.6]. We prove the pro version of the acyclicity by using a totally different method; it is closer to the methods developed in [Su82, Su95].

In $\S 1$, we prove the pro stability for $H_{1}\left(\mathrm{GL}_{n}\right)$, cf. Theorem 1.5. This essentially follows from Vaseršteřn's stability for relative $K_{1}$. In $\S 2$, we recall some properties of Tor-unital rings, which we need later. $\S 3$ is the technical heart of this paper. In this section, we study triangular spaces and prove a pro acyclicity of the union of triangular spaces, cf. Theorem 3.9. In $\S 4$, we complete the proof of Theorem 0.3 , using the pro acyclicity of triangular spaces.

\footnotetext{
1"commutative" means that each $A_{m}$ is commutative. However, this condition may not be essential. We expect that the theorem is true without the commutativity assumption.
} 


\subsection{Notation.}

1. A ring means an associative, not necessarily unital, ring.

2. $\operatorname{sr}(A)$ is the stable range of a ring $A$, i.e. the minimum number $r \geq 1$ such that the stable range condition [Va69, $(2.2)_{n}$ ] holds for every $n \geq r$.

3. Let $A$ be a ring and $n \geq 1$.

(a) The general linear group $\mathrm{GL}_{n}(A)$ is the kernel of the canonical map $\mathrm{GL}_{n}(\mathbb{Z} \ltimes A) \rightarrow \mathrm{GL}_{n}(\mathbb{Z})$.

(b) The elementary subgroup $E_{n}(A)$ is the subgroup of $\mathrm{GL}_{n}(A)$ generated by the elementary matrices $e_{i j}(a)$ with $a \in A$ and $1 \leq i \neq j \leq n$.

We regard $\mathrm{GL}_{n}(A)$ as a subgroup of $\mathrm{GL}_{n+1}(A)$ by sending a matrix $\alpha$ to $\left(\begin{array}{cc}\alpha & 0 \\ 0 & 1\end{array}\right)$. We write $\operatorname{GL}(A)=$ $\mathrm{GL}_{\infty}(A)=\bigcup_{n} \mathrm{GL}_{n}(A)$ and $E(A)=E_{\infty}(A)=\bigcup_{n} E_{n}(A)$.

4. A pro ring is a pro system of rings indexed by a filtered poset. Typically, we denote a pro ring by a bold letter $\mathbf{A}=\left\{A_{m}\right\}$ and the structured maps $A_{m} \rightarrow A_{n}$ by $\iota_{m, n}$ or just by $\iota$.

5. A unital (resp. commutative) pro ring is a pro ring which is levelwise unital (resp. commutative). Unless otherwise stated, we use standard operations of rings levelwise for pro rings: E.g. $\mathrm{GL}_{n}(\mathbf{A})=$ $\left\{\mathrm{GL}_{n}\left(A_{m}\right)\right\}_{m}, \operatorname{Tor}_{*}^{\mathbb{Z} \ltimes \mathbf{A}}(\mathbb{Z}, \mathbb{Z})=\left\{\operatorname{Tor}_{*}^{\mathbb{Z} \ltimes A_{m}}(\mathbb{Z}, \mathbb{Z})\right\}_{m}$, etc.

6. A left ideal of a pro ring $\mathbf{A}=\left\{A_{m}\right\}_{m \in J}$ is a pro ring $\mathbf{B}=\left\{B_{m}\right\}_{m \in J}$ with a level map $\mathbf{B} \rightarrow \mathbf{A}$ which exhibits each $B_{m}$ as a left ideal of $A_{m}$.

0.4. Acknowledgment. The study of homology pro stability has started when I was working on relative Chern classes with Wataru Kai [IK17]. I am very grateful to him for many discussions and useful comments. The majority of this paper was written when I was visiting Moritz Kerz in Universität Regensburg. I thank him for his kind hospitality and interest in my work. This work was supported by JSPS KAKENHI Grant Number 16J08843, and by the Program for Leading Graduate Schools, MEXT, Japan. 


\section{Pro stability for $K_{1}$}

1.1. Vaserště̆n's stability. Let $R$ be a unital ring and $A$ a two-sided ideal of $R$. The normal elementary subgroup $E_{n}(R, A)$ is the smallest normal subgroup of $E_{n}(R)$ which contains $E_{n}(A)$. We write $E(R, A)=E_{\infty}(R, A)=\bigcup_{n} E_{n}(R, A)$. By Whitehead's lemma, $E(R, A)$ is a normal subgroup of $\mathrm{GL}(A)$. We define the relative $K_{1}$-group $K_{1}(R, A)$ to be the quotient group $\operatorname{GL}(A) / E(R, A)$.

Theorem 1.1 (Vaseršteĭn [Va69]). The canonical map

$$
\mathrm{GL}_{n}(A) \rightarrow K_{1}(R, A)
$$

is surjective for $n \geq \operatorname{sr}(A)$, and the kernel is $E_{n}(R, A)$ for $n \geq \operatorname{sr}(A)+1$.

1.2. Let $R$ be a unital ring and $A$ a two-sided ideal of $R$. The following lemma generalizes [Ti76, ??] for noncommutative rings.

Lemma 1.2. For $n \geq 3, E_{n}\left(R, A^{2}\right) \subset\left[E_{n}(A), E_{n}(A)\right]$.

Proof. Note the standard equality of elementary matrices;

$$
\left[e_{i j}(a), e_{k l}(b)\right]= \begin{cases}1 & \text { if } j \neq k, i \neq l \\ e_{i l}(a b) & \text { if } j=k, i \neq l \\ e_{k j}(-b a) & \text { if } j \neq k, i=l\end{cases}
$$

which we use throughout the proof. One immediate consequence is that $E_{n}\left(A^{2}\right) \subset\left[E_{n}(A), E_{n}(A)\right]$ for $n \geq 3$.

For $r=\left(r_{1}, \ldots, r_{n}\right) \in R^{n}$ with $r_{j}=1$, we write

$$
X_{j}(r):=\prod_{k \neq j} e_{j k}\left(r_{k}\right) \quad \text { and } \quad X^{j}(r):=\prod_{k \neq j} e_{k j}\left(r_{k}\right) .
$$

Fix $1 \leq j \leq n$. It is easy to see that every $x \in E_{n}(R)$ has the form

$$
x_{2 m}(U):=X^{j}\left(u_{2 m}\right) X_{j}\left(u_{2 m-1}\right) \cdots X^{j}\left(u_{2}\right) X_{j}\left(u_{1}\right)
$$

for some $m>0$ and $U=\left(u_{1}, u_{2} \ldots, u_{2 m}\right) \in\left(R^{n}\right)^{2 m}$. We also set $x_{0}(\emptyset):=1$ and

$$
x_{2 m-1}(V):=X^{j}\left(v_{2 m-1}\right) X_{j}\left(v_{2 m-2}\right) \cdots X_{j}\left(v_{2}\right) X^{j}\left(v_{1}\right)
$$

for $m>0$ and $V=\left(v_{1}, v_{2} \ldots, v_{2 m-1}\right) \in\left(R^{n}\right)^{2 m-1}$.

Consider the following assertion.

$(\varnothing)_{N}$ For every $U \in\left(R^{n}\right)^{N}, x_{N}(U) E_{n}\left(A^{2}\right) x_{N}(U)^{-1} \subset\left[E_{n}(A), E_{n}(A)\right]$.

We have seen $(\nabla)_{0}$. Let $N>0$ and suppose that $(\nabla)_{l}$ holds for $l<N$. We shall prove $(\nabla)_{N}$ in case $N$ even; the case $N$ odd is proved in the same way.

Let $U=\left(u_{1}, \ldots, u_{N}\right) \in\left(R^{n}\right)^{N}$ and $x:=x_{N}(U)$. For $e_{i k}(a)$ with $a \in A^{2}, 1 \leq i, k \leq n$ and $k \neq j$, we have $X_{j}\left(u_{1}\right) e_{i k}(a) X_{j}\left(-u_{1}\right) \in E_{n}\left(A^{2}\right)$ and thus by the induction hypothesis $x e_{i k}(a) x^{-1} \in$ $\left[E_{n}(A), E_{n}(A)\right]$. For $e_{i j}(a)$ with $a \in A^{2}$ and $1 \leq i \neq j \leq n$, we have

$$
\begin{aligned}
X_{j}\left(u_{1}\right) e_{i j}(a) X_{j}\left(-u_{1}\right) & =e_{j i}\left(u_{1, i}\right)\left(\prod_{k \neq i, j} e_{i k}\left(-a u_{1, k}\right) \cdot e_{i j}(a)\right) e_{j i}\left(-u_{1, i}\right) \\
& =\prod_{k \neq i, j} e_{j k}\left(-u_{1, i} a u_{1, k}\right) e_{i k}\left(-a u_{1, k}\right) \cdot e_{j i}\left(u_{1, i}\right) e_{i j}(a) e_{j i}\left(-u_{1, i}\right) .
\end{aligned}
$$

Hence, it follows from the induction hypothesis that $x E_{n}\left(A^{2}\right) x^{-1}$ is generated by $y_{i} e_{i j}(a) y_{i}^{-1}, y_{i}=$ $X^{j}\left(u_{N}\right) X_{j}\left(u_{N-1}\right) \cdots X^{j}\left(u_{2}\right) e_{j i}\left(u_{1, i}\right)$, with $a \in A^{2}$ and $1 \leq i \neq j \leq n$ modulo $\left[E_{n}(A), E_{n}(A)\right]$.

For $U=\left(u_{1}, \ldots, u_{N}\right) \in\left(R^{n}\right)^{N}$ and $1 \leq p \leq N / 2$, we set

$$
\begin{gathered}
y_{i}^{2 p-1}(U):=X^{j}\left(u_{N}\right) X_{j}\left(u_{N-1}\right) \cdots X^{j}\left(u_{2 p}\right) e_{j i}\left(u_{2 p-1, i}\right) \cdots e_{i j}\left(u_{2, i}\right) e_{j i}\left(u_{1, i}\right) \\
y_{i}^{2 p}(U):=X^{j}\left(u_{N}\right) X_{j}\left(u_{N-1}\right) \cdots X_{j}\left(u_{2 p+1}\right) e_{i j}\left(u_{2 p, i}\right) \cdots e_{i j}\left(u_{2, i}\right) e_{j i}\left(u_{1, i}\right) .
\end{gathered}
$$

We claim that: 
$(\diamond)_{Q}$ For $U \in\left(R^{n}\right)^{N}, x_{N}(U) E_{n}\left(A^{2}\right) x_{N}(U)^{-1}$ is generated by $y_{i}^{Q}(U) e_{i j}(a) y_{i}^{Q}(U)^{-1}, a \in A^{2}$, $1 \leq i \neq j \leq n$ modulo $\left[E_{n}(A), E_{n}(A)\right]$.

We have seen $(\diamond)_{1}$. Let $Q>1$ and suppose that $(\diamond)_{l}$ holds for $l<Q$. We prove $(\diamond)_{Q}$ in case $Q$ even; the case $Q$ odd is proved in the same way.

Let $U=\left(u_{1}, \ldots, u_{N}\right) \in\left(R^{n}\right)^{N}$. According to $(\diamond)_{Q-1}, x_{N}(U) E_{n}\left(A^{2}\right) x_{N}(U)^{-1}$ is generated by $y_{i}^{Q-1}(U) e_{i j}(a) y_{i}^{Q-1}(U)^{-1}, a \in A^{2}, 1 \leq i \neq j \leq n$ modulo $\left[E_{n}(A), E_{n}(A)\right]$. We fix $1 \leq i \neq j \leq n$ for a moment. Now,

$$
X^{j}\left(u_{Q}\right) e_{j i}\left(u_{Q-1, i}\right)=e_{i j}\left(u_{Q, i}\right) e_{j i}\left(u_{Q-1, i}\right) \prod_{k \neq i, j} e_{k j}\left(u_{Q, k}\right) e_{k i}\left(u_{Q, k} u_{Q-1, i}\right) .
$$

Hence, by putting $\tilde{y}:=\prod_{k \neq i, j} e_{k i}\left(u_{2 p, k} u_{2 p-1, i}\right)$, we have

$$
y_{i}^{Q-1}(U)=X^{j}\left(u_{N}\right) X_{j}\left(u_{N-1}\right) \cdots X_{j}\left(u_{Q+1}\right) e_{i j}\left(u_{Q, i}\right) e_{j i}\left(u_{Q-1, i}\right) X^{j}\left(u_{Q-2}^{\prime}\right) \cdots X^{j}\left(u_{2}^{\prime}\right) X_{j}\left(u_{1}^{\prime}\right) \tilde{y}
$$

for some $u_{1}^{\prime}, \ldots, u_{Q-2}^{\prime} \in R^{n}$ with $u_{q, i}^{\prime}=u_{q, i}$. For $Q-1 \leq q \leq N$, we set

$$
u_{q}^{\prime}:= \begin{cases}u_{q, i} e_{i}+e_{j} & \text { if } q=Q-1, Q \\ u_{q} & \text { if } q>Q\end{cases}
$$

and $U^{\prime}:=\left(u_{1}^{\prime}, \ldots, u_{N}^{\prime}\right)$, so that $y_{i}^{Q-1}(U)=x_{N}\left(U^{\prime}\right) \tilde{y}$ and $y_{i}^{q}\left(U^{\prime}\right)=y_{i}^{q}(U)$ for $q \geq Q$. By applying $(\diamond)_{Q-1}$ for $U^{\prime}$, we see that $x_{N}\left(U^{\prime}\right) E_{n}\left(A^{2}\right) x_{N}\left(U^{\prime}\right)^{-1}$ is generated by $y_{i}^{Q}\left(U^{\prime}\right) e_{i j}(a) y_{i}^{Q}\left(U^{\prime}\right)^{-1}, a \in A^{2}$ modulo $\left[E_{n}(A), E_{n}(A)\right]$. Varying $i$, this proves $(\diamond)_{Q}$ for the given $U \in\left(R^{n}\right)^{N}$, and thus for all $U \in$ $\left(R^{n}\right)^{N}$.

According to $(\diamond)_{N}$, to prove $(\nabla)_{N}$, it suffices to show that $y e_{i j}(a b) y^{-1} \in\left[E_{n}(A), E_{n}(A)\right]$ for $y=$ $e_{i j}\left(r_{N}\right) e_{j i}\left(r_{N-1}\right) \cdots e_{i j}\left(r_{2}\right) e_{j i}\left(r_{1}\right)$ with $a, b \in A, r_{1}, \ldots, r_{N} \in R$ and $1 \leq i \neq j \leq n$. Observe that we have

$$
\begin{aligned}
e_{i j}\left(r_{1}\right) e_{j i}(a b) e_{i j}\left(-r_{1}\right) & =e_{i j}\left(r_{1}\right)\left[e_{j t}(a), e_{t i}(b)\right] e_{i j}\left(-r_{1}\right) \\
& =\left[e_{i t}\left(r_{1} a\right) e_{j t}(a), e_{t j}\left(-b r_{1}\right) e_{t i}(b)\right]
\end{aligned}
$$

for $t \neq i, j$. Now, it is clear that $y^{\prime}\left[e_{i t}\left(r_{1} a\right) e_{j t}(a), e_{t j}\left(-b r_{1}\right) e_{t i}(b)\right]\left(y^{\prime}\right)^{-1} \in\left[E_{n}(A), E_{n}(A)\right]$ for $y^{\prime}=$ $e_{i j}\left(r_{N}\right) e_{j i}\left(r_{N-1}\right) \cdots e_{i j}\left(r_{2}\right)$, and thus we get $(\Omega)_{N}$.

Corollary 1.3. Let $\mathbf{R}=\left\{R_{m}\right\}$ be a unital pro ring and $\mathbf{A}=\left\{A_{m}\right\}$ a two-sided ideal of $\mathbf{R}$. Suppose that $\mathbf{A} / \mathbf{A}^{2}=\left\{A_{m} / A_{m}^{2}\right\}=0$. Then, for $3 \leq n \leq \infty$, the canonical maps

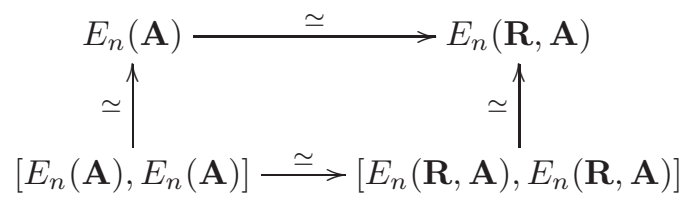

are pro isomorphisms.

Proof. Since all the indicated maps are injections, it suffices to show that the map $\left[E_{n}(\mathbf{A}), E_{n}(\mathbf{A})\right] \rightarrow$ $E_{n}(\mathbf{R}, \mathbf{A})$ is a pro epimorphism. By the assumption $\mathbf{A} / \mathbf{A}^{2}=0$, there exists $s \geq m$ for each $m$ such that $\iota_{s, m}\left(A_{s}\right) \subset A_{m}^{2}$. Therefore,

$$
\iota_{s, m} E_{n}\left(R_{s}, A_{s}\right) \subset E_{n}\left(R_{m}, A_{m}^{2}\right) \subset\left[E_{n}\left(R_{m}, A_{m}\right), E_{n}\left(R_{m}, A_{m}\right)\right],
$$

where the last inclusion is by Lemma 1.2. This proves that $\left[E_{n}(\mathbf{A}), E_{n}(\mathbf{A})\right] \rightarrow E_{n}(\mathbf{R}, \mathbf{A})$ is a pro epimorphism. 
1.3. Pro excision and pro stability. Let $\mathbf{R}=\left\{R_{m}\right\}$ be a unital pro ring and $\mathbf{A}=\left\{A_{m}\right\}$ a two-sided ideal of $\mathbf{R}$. We define $\operatorname{sr}(\mathbf{A}):=\max _{m}\left(\operatorname{sr}\left(A_{m}\right)\right)$.

Theorem 1.4 (Pro exision). Suppose that $\mathbf{A} / \mathbf{A}^{2}=0$. Then the canonical map

$$
H_{1}(\mathrm{GL}(\mathbf{A})) \stackrel{\sim}{\rightarrow} K_{1}(\mathbf{R}, \mathbf{A})
$$

is a pro isomorphism.

Proof. Since $K_{1}(\mathbf{R}, \mathbf{A})$ is levelwise abelian, we have a levelwise exact sequence

$$
H_{1}(E(\mathbf{R}, \mathbf{A})) \longrightarrow H_{1}(\mathrm{GL}(\mathbf{A})) \longrightarrow K_{1}(\mathbf{R}, \mathbf{A}) \longrightarrow 0 \text {. }
$$

According to Corollary 1.3, $H_{1}(E(\mathbf{R}, \mathbf{A}))=0$, and thus we get $H_{1}(\mathrm{GL}(\mathbf{A})) \simeq K_{1}(\mathbf{R}, \mathbf{A})$.

Theorem 1.5 (Pro stability). Suppose that $\mathbf{A} / \mathbf{A}^{2}=0$. Then the canonical map

$$
H_{1}\left(\mathrm{GL}_{n}(\mathbf{A})\right) \rightarrow H_{1}(\mathrm{GL}(\mathbf{A}))
$$

is a pro epimorphism for $n \geq \operatorname{sr}(\mathbf{A})$ and a pro isomorphism for $n \geq \max (3, \operatorname{sr}(\mathbf{A})+1)$.

Proof. The composite

$$
H_{1}\left(\mathrm{GL}_{n}(\mathbf{A})\right) \rightarrow H_{1}(\mathrm{GL}(\mathbf{A})) \stackrel{\sim}{\rightarrow} K_{1}(\mathbf{R}, \mathbf{A})
$$

is a levelwise surjection for $n \geq \operatorname{sr}(\mathbf{A})$ by Theorem 1.1. Since the last map is a pro isomorphism by Theorem 1.4, the first map is a pro epimorphism for $n \geq \operatorname{sr}(\mathbf{A})$.

Consider the commutative diagram

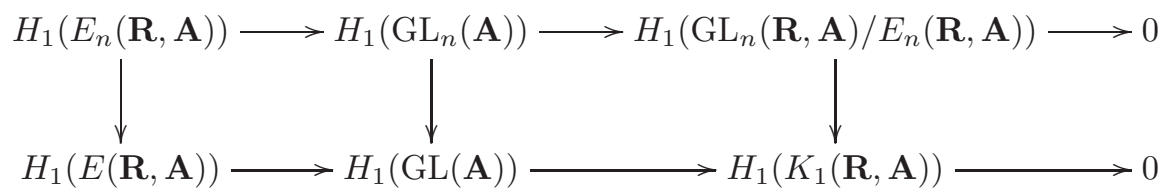

with levelwise exact rows. The left terms are zero for $n \geq 3$ by Corollary 1.3. According to Theorem 1.1, the right vertical map is a levelwise bijection for $n \geq \operatorname{sr}(\mathbf{A})+1$. Hence, the middle term is a pro isomorphism for $n \geq \max (3, \operatorname{sr}(\mathbf{A})+1)$.

Theorem 1.6. Set $\bar{E}_{n}(\mathbf{A}):=\mathrm{GL}_{n}(\mathbf{A}) \cap E(\mathbf{A})$. Suppose that $\mathbf{A} / \mathbf{A}^{2}=0$. Then the canonical map

$$
E_{n}(\mathbf{A}) \rightarrow \bar{E}_{n}(\mathbf{A})
$$

is a pro isomorphism for $n \geq \max (3, \operatorname{sr}(\mathbf{A})+1)$.

Proof. Let $\bar{E}_{n}(\mathbf{R}, \mathbf{A}):=\mathrm{GL}_{n}(\mathbf{A}) \cap E(\mathbf{R}, \mathbf{A})$. According to Theorem 1.1, the canonical map $E_{n}(\mathbf{R}, \mathbf{A}) \rightarrow$ $\bar{E}_{n}(\mathbf{R}, \mathbf{A})$ is a levelwise bijection for $n \geq \operatorname{sr}(\mathbf{A})+1$. Hence, the theorem follows from Corollary 1.3. 


\section{TOR-UNITAL PRO RINGS}

The treatment of this section closely follows Suslin [Su95] and Geisser-Hesselholt [GH06].

\subsection{Definitions.}

Definition 2.1. A pro ring $\mathbf{A}=\left\{A_{m}\right\}$ is Tor-unital if

$$
\operatorname{Tor}_{i}^{\mathbb{Z} \ltimes \mathbf{A}}(\mathbb{Z}, \mathbb{Z})=\left\{\operatorname{Tor}_{i}^{\mathbb{Z} \ltimes A_{m}}(\mathbb{Z}, \mathbb{Z})\right\}_{m}=0
$$

as pro abelian groups for all $i>0$.

Example 2.2.

(i) A unital pro ring, i.e. a pro system of unital rings, is Tor-unital.

(ii) (Morrow [Mo15]) Let $A$ be an ideal of a noetherian commutative ring, then $\left\{A^{m}\right\}_{m \geq 1}$ is Tor-unital.

Definition 2.3. Let $\mathbf{A}=\left\{A_{m}\right\}_{m \in J}$ be a pro ring.

(i) A left $\mathbf{A}$-module is a pro abelian group $\mathbf{M}=\left\{M_{m}\right\}_{m \in J}$ with a level map $\mathbf{A} \times \mathbf{M} \rightarrow \mathbf{M}$ which exhibits each $M_{m}$ as a left $A_{m}$-module. A morphism between left $\mathbf{A}$-modules $\mathbf{M}=\left\{M_{m}\right\}$ and $\mathbf{N}=\left\{N_{m}\right\}$ is a level map $f: \mathbf{M} \rightarrow \mathbf{N}$ such that each $f_{m}: M_{m} \rightarrow N_{m}$ is a morphism of left $A_{m}$-modules.

(ii) A left $\mathbf{A}$-module $\mathbf{P}$ is pseudo-free if there is an isomorphism of left $\mathbf{A}$-modules $\mathbf{A} \otimes \mathbf{L} \stackrel{\simeq}{\longrightarrow}$ for some pro system $\mathbf{L}$ of free abelian groups. We call such an $\mathbf{L}$ a free basis of $\mathbf{P}$.

(iii) A morphism $f: \mathbf{P} \rightarrow \mathbf{M}$ of left $\mathbf{A}$-modules is special if $\mathbf{P}$ is pseudo-free with a free basis $\mathbf{L}$ and $f$ is induced from a level morphism of pro abelian groups $\mathbf{L} \rightarrow \mathbf{M}$.

\subsection{Pro resolution.}

Proposition 2.4 (Suslin [Su95], Geisser-Hesselholt [GH06]). Let $\mathbf{A}=\left\{A_{m}\right\}$ be a Tor-unital pro ring. Suppose we are given an augmented complex

$$
\ldots \rightarrow \mathbf{C}_{1} \rightarrow \mathbf{C}_{0} \stackrel{\epsilon}{\rightarrow} \mathbf{C}_{-1}
$$

of left A-modules such that: ${ }^{2}$

(i) Each $\mathbf{C}_{k}$ with $k \geq-1$ is pseudo-free.

(ii) The homology $H_{k}\left(C_{\bullet}, m\right)$ is annihilated by $A_{m}$ for every $m$ and $k \geq-1$.

Then

for all $k \geq-1$.

$$
H_{k}\left(\mathbf{C}_{\bullet}\right)=\left\{H_{k}\left(C_{\bullet}, m\right)\right\}_{m}=0
$$

In fact, a finer result holds.

Proposition 2.5. Let $\mathbf{A}=\left\{A_{m}\right\}_{m \in J}$ be a Tor-unital pro ring and $k \geq-1$. Then there exists $s(m) \geq m$ for each $m \in J$ such that the map

$$
\iota_{s(m), m}: H_{k}\left(C_{\bullet}, s(m)\right) \rightarrow H_{k}\left(C_{\bullet}, m\right)
$$

is zero for all augmented complexes of left $\mathbf{A}$-modules which satisfy the conditions (i) (ii).

Proof. Let $\mathbf{C}$ be a pseudo-free left $\mathbf{A}$-module with a free basis $\mathbf{L}$. Then we have levelwise isomorphisms

$$
\begin{aligned}
\operatorname{Tor}_{q}^{\mathbb{Z} \ltimes \mathbf{A}}(\mathbb{Z}, \mathbf{C}) & \simeq \operatorname{Tor}_{q}^{\mathbb{Z} \ltimes \mathbf{A}}(\mathbb{Z}, \mathbf{A} \otimes \mathbf{L}) \\
& \simeq \operatorname{Tor}_{q}^{\mathbb{Z} \ltimes \mathbf{A}}(\mathbb{Z}, \mathbf{A}) \otimes \mathbf{L} \\
& \simeq \operatorname{Tor}_{q+1}^{\mathbb{Z} \ltimes \mathbf{A}}(\mathbb{Z}, \mathbb{Z}) \otimes \mathbf{L} .
\end{aligned}
$$

Since $\mathbf{A}$ is Tor-unital, we see that

$$
\operatorname{Tor}_{q}^{\mathbb{Z} \ltimes \mathbf{A}}(\mathbb{Z}, \mathbf{C})=0
$$

for every $q \geq 0$.

\footnotetext{
${ }^{2}$ We thank Takeshi Saito for pointing out an unnecessary condition, the augmentation $\epsilon$ is special, which was in the first draft and in [Su95, GH06] too.
} 
Let $\mathbf{Z}_{k}$ and $\mathbf{B}_{k-1}$ are the kernel and the image of $\mathbf{C}_{k} \rightarrow \mathbf{C}_{k-1}$ respectively. By the assumption (ii), we have a levelwise inclusion $\mathbf{A C}_{-1} \subset \mathbf{B}_{-1}$, and thus there is a levelwise surjection $\mathbf{C}_{-1} / \mathbf{A C}_{-1} \rightarrow$ $H_{-1}\left(\mathbf{C}_{\bullet}\right)$. Since $\mathbf{C}_{-1}$ is pseudo-free, $\mathbf{C}_{-1} / \mathbf{A} \mathbf{C}_{-1}=\operatorname{Tor}_{0}^{\mathbb{Z} \ltimes \mathbf{A}}\left(\mathbb{Z}, \mathbf{C}_{-1}\right)=0$. Therefore, $H_{-1}\left(\mathbf{C}_{\bullet}\right)=0$.

Let $k \geq 0$ and suppose that $H_{l}\left(\mathbf{C}_{\bullet}\right)=0$ for $l<k$. Consider the levelwise spectral sequence

$$
\mathbf{E}_{p q}^{1}= \begin{cases}\operatorname{Tor}_{q}^{\mathbb{Z} \ltimes \mathbf{A}}\left(\mathbb{Z}, \mathbf{C}_{p}\right) & \text { if } 0 \leq p \leq k \\ \operatorname{Tor}_{q}^{\mathbb{Z} \ltimes \mathbf{A}}\left(\mathbb{Z}, \mathbf{Z}_{k}\right) & \text { if } p=k+1 \\ 0 & \text { otherwise, }\end{cases}
$$

which arises from the brutal truncation of the complex

$$
\mathbf{Z}_{k} \rightarrow \mathbf{C}_{k} \rightarrow \mathbf{C}_{k-1} \rightarrow \ldots \rightarrow \mathbf{C}_{0}
$$

By the induction hypothesis, the complex is pro quasi-isomorphic to $\mathbf{C}_{-1}$ and thus

$$
\mathbf{E}_{q}^{\infty} \simeq \operatorname{Tor}_{q}^{\mathbb{Z} \ltimes \mathbf{A}}\left(\mathbb{Z}, \mathbf{C}_{-1}\right)=0
$$

for $q \geq 0$. Since $\mathbf{C}_{p}$ is pseudo-free, we also have $\mathbf{E}_{p q}^{1}=0$ for $0 \leq p \leq k$. Hence,

$$
\mathbf{Z}_{k} / \mathbf{A} \mathbf{Z}_{k}=\operatorname{Tor}_{0}^{\mathbb{Z} \ltimes \mathbf{A}}\left(\mathbb{Z}, \mathbf{Z}_{k}\right)=\mathbf{E}_{k+1}^{\infty}=0 .
$$

On the other hand, by the assumption (ii), we have $\mathbf{A} \mathbf{Z}_{k} \subset \mathbf{B}_{k}$. Therefore, $H_{k}\left(\mathbf{C}_{\bullet}\right)=0$.

A finer variant is also clear from this proof.

Lemma 2.6. Let $\mathbf{A}$ be a pro ring and $\mathbf{P}$ a pseudo-free left $\mathbf{A}$-module. Then there exists an augmented complex $\mathbf{P}$. of left $\mathbf{A}$-modules with $\mathbf{P}_{-1}=\mathbf{P}$ which satisfies the conditions ( $i$ ) (ii) and

(iii) The augmentation $\epsilon: \mathbf{P}_{0} \rightarrow \mathbf{P}_{-1}$ is special

We call $\mathbf{P}$. a pro resolution of $\mathbf{P}$.

Proof. Write $\mathbf{P}=\left\{P_{m}\right\}$ and let $\mathbb{Z}[\mathbf{P}]=\left\{\mathbb{Z}\left[P_{m}\right]\right\}$ be the pro system of the free abelian groups generated by the sets $P_{m}$. Then $\mathbf{P}_{0}:=\mathbf{A} \otimes \mathbb{Z}[\mathbf{P}]$ is a pseudo-free $\mathbf{A}$-module and the canonical map $\mathbb{Z}[\mathbf{P}] \rightarrow \mathbf{P}$ induces a special morphism $\epsilon: \mathbf{P}_{0} \rightarrow \mathbf{P}$.

Let $\mathbf{R}=\left\{R_{m}\right\}$ be the kernel of $\epsilon$, and $\mathbb{Z}[\mathbf{R}]=\left\{\mathbb{Z}\left[R_{m}\right]\right\}$ the pro system of the free abelian group generated by $R_{m}$. Then $\mathbf{P}_{1}:=\mathbf{A} \otimes \mathbb{Z}[\mathbf{R}]$ is a pseudo-free $\mathbf{A}$-module. Repeating this procedure, we obtain an augmented complex $\mathbf{P}$. with $\mathbf{P}_{-1}=\mathbf{P}$ which satisfies the desired conditions. 


\section{Pro aCyClicity of triangular SPACES}

The goal of this section is to prove Theorem 3.9.

\subsection{Preliminaries on homology.}

3.1.1. For a simplicial set $X$, we denote by $C_{*}(X)$ the complex freely generated by $X_{*}$ with the differential being the alternating sum of the faces. We write $H_{n}(X)=H_{n}\left(C_{*}(X)\right)$. Also, we write $\tilde{H}_{n}(X)$ for the reduced homology.

Let $G$ be a group. We write $E G$ for the simplicial set whose degree $n$-part is $G^{\times(n+1)}$ and whose $i$-th face (resp. the $i$-th degeneracy) omits the $i$-th entry (resp. repeats the $i$-th entry). We give a right $G$-action on $E G$ by $\left(g_{0}, \ldots, g_{n}\right) \cdot g:=\left(g_{0} g, \ldots, g_{n} g\right)$. The classifying space $B G$ is defined to be $E G / G$.

3.1.2. By a pro object or pro system, we mean a pro object whose index category is a left filtered small category.

Lemma 3.1. Let $f: X \rightarrow Y$ be a morphism between pro systems of pointed simplicial sets. Suppose that $f$ induces pro isomorphisms

$$
\pi_{n}(X) \stackrel{\sim}{\longrightarrow} \pi_{n}(Y)
$$

for all $n \geq 0$. Then $f$ induces pro isomorphisms

$$
H_{n}(X) \stackrel{\sim}{\rightarrow} H_{n}(Y)
$$

for all $n \geq 0$.

Proof. Since $\mathbb{Z} \pi_{0}(X) \simeq H_{0}(X)$, the assertion is clear for $n=0$. Hence, by taking the connected components, we may assume that $X$ and $Y$ are connected.

Then, according to [Is01], for each $n \geq 1$, the induced map $P_{n}(X) \rightarrow P_{n}(Y)$ on the $n$-th Postnikov sections is a strict weak equivalence, i.e. isomorphic to a levelwise weak equivalence. Hence, the induced map $C_{*}\left(P_{n}(X)\right) \rightarrow C_{*}\left(P_{n}(Y)\right)$ is isomorphic to a levelwise quasi-isomorphism.

On the other hand, by Hurewicz theorem and Serre spectral sequence, we have levelwise isomorphisms $H_{k}(X) \simeq H_{k}\left(P_{n}(X)\right)$ for $k \leq n$. Now, in the commutative diagram

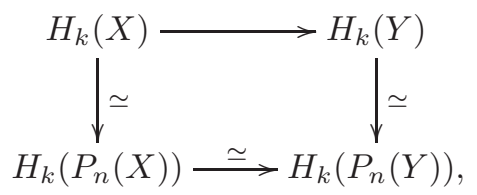

the vertical maps and the bottom map are pro isomorphisms for $k \leq n$, and so is the top map. Since $n$ is arbitrary, $H_{k}(X) \stackrel{\simeq}{\rightarrow} H_{k}(Y)$ is a pro isomorphism for every $k \geq 0$.

For a simplicial group $G$, we consider the bi-simplicial set $B G$ constructed degreewise. For a bisimplicial set $X$, we denote by $C_{*}(X)$ the double-complex freely generated by $X_{*}$ with the differential being the alternating sum of the faces.

Corollary 3.2. Let $f: P \rightarrow Q$ be a morphism between pro systems of simplicial abelian groups. Suppose that $f$ induces pro isomorphisms

$$
\pi_{n}(P) \stackrel{\sim}{\longrightarrow} \pi_{n}(Q)
$$

for all $n \geq 0$. Then $f$ induces pro isomorphisms

$$
H_{n}\left(\operatorname{Tot} C_{*}(B P)\right) \stackrel{\sim}{\rightarrow} H_{n}\left(\operatorname{Tot} C_{*}(B Q)\right)
$$

for all $n \geq 0$.

Proof. Now, the morphism $B_{k} P \rightarrow B_{k} Q$ induces pro isomorphisms $\pi_{n}\left(B_{k} P\right) \rightarrow \pi_{n}\left(B_{k} Q\right)$ for all $n \geq 0$. Hence, by Lemma 3.1, the induced maps

$$
H_{n}\left(C_{*}\left(B_{k} P\right)\right) \rightarrow H_{n}\left(C_{*}\left(B_{k} Q\right)\right)
$$

are pro isomorphisms for all $n \geq 0$. By the standard spectral sequence, we obtain the corollary. 
3.1.3. Let us quote a lemma from $[\mathrm{Su} 95, \S 2]$.

Lemma 3.3. Let $G$ be a group and $H$ a group with a left $G$-action. Then there exists a natural quasiisomorphism

$$
C_{*}(B(G \ltimes H)) \simeq C_{*}(E G) \otimes_{G} C_{*}(B H) .
$$

Let $G=\left\{G_{m}\right\}$ be a pro group (= a pro system of groups). A left $G$-module $M$ is a pro abelian group $M=\left\{M_{m}\right\}$ with a level map $G \times M \rightarrow M$ which exhibits each $M_{m}$ as a left $G_{m}$-module. A morphism between left $G$-modules $M=\left\{M_{m}\right\}$ and $N=\left\{N_{m}\right\}$ is a level map $f: M \rightarrow N$ such that each $f_{m}: M_{m} \rightarrow N_{m}$ is a morphism of left $G_{m}$-modules. These form the category of left $G$-modules, and we consider simplicial objects in this category; simplicial left $G$-modules and morphisms between them.

Corollary 3.4. Let $G$ be a pro group. Let $P$ and $Q$ be simplicial left $G$-modules and $f: P \rightarrow Q$ a morphism between them. Suppose that $f$ induces pro isomorphisms

$$
\pi_{n}(P) \stackrel{\sim}{\longrightarrow} \pi_{n}(Q)
$$

for all $n \geq 0$. Then $f$ induces pro isomorphisms

$$
H_{n}\left(\operatorname{Tot} C_{*}(B(G \ltimes P))\right) \stackrel{\sim}{\longrightarrow} H_{n}\left(\operatorname{Tot} C_{*}(B(G \ltimes Q))\right)
$$

for all $n \geq 0$, where the semi-direct products are taken levelwise and degreewise.

Proof. This follows from Corollary 3.2 and Lemma 3.3.

\subsection{The key lemma.}

3.2.1. Triangular spaces. Let $A$ be a ring and $P$ a left $A$-module. Let $\sigma$ be a finite poset. We define a group $T^{\sigma}(A, P)$ by

$$
T^{\sigma}(A, P):=\prod_{i<\sigma j, j \notin \max \sigma} A_{(i, j)} \times \prod_{i<\sigma j, j \in \max \sigma} P_{(i, j)},
$$

where $A_{(i, j)}$ and $P_{(i, j)}$ are just the copies of $A$ and $P$ respectively. For $\alpha \in T^{\sigma}(A, P)$, we denote its $(i, j)$ th component by $\alpha_{i, j}$; thus $\alpha_{i, j} \in A$ if $j \notin \max \sigma$, and $\alpha_{i, j} \in P$ if $j \in \max \sigma$. For $\alpha, \beta \in T^{\sigma}(A, P)$, we define the composition $\alpha \cdot \beta$ by

$$
(\alpha \cdot \beta)_{i, j}=\alpha_{i, j}+\beta_{i, j}+\sum_{i<\sigma k<_{\sigma} j} \alpha_{i, k} \beta_{k, j}
$$

for $i<_{\sigma} j$. We shorten $T^{\sigma}(A):=T^{\sigma}(A, A)$.

Set $\sigma_{0}:=\sigma \backslash \max \sigma$. Then we have an identification

$$
T^{\sigma}(A, P)=T^{\sigma_{0}}(A) \ltimes M_{\sigma_{0}, \max \sigma}(P),
$$

and canonical inclusion and projection

$$
T^{\sigma_{0}}(A) \hookrightarrow T^{\sigma}(A, P) \rightarrow T^{\sigma_{0}}(A) .
$$

Let $\theta: \sigma \rightarrow \tau$ be a morphism of finite posets. Then it induces a morphism of groups

$$
T^{\theta}: T^{\sigma}(A) \rightarrow T^{\tau}(A) .
$$

If $\theta^{-1}(\max \tau)=\max (\sigma)$, then it also induces a morphism $T^{\sigma}(A, P) \rightarrow T^{\tau}(A, P)$ for any left $A$-module $P$, which we also denote by $T^{\theta}$.

Let $f: P \rightarrow Q$ be a morphism of $A$-modules. Then it induces a morphism of groups

$$
T^{f}: T^{\sigma}(A, P) \rightarrow T^{\sigma}(A, Q)
$$

If $\theta: \sigma \rightarrow \tau$ satisfies $\theta^{-1}(\max \tau)=\max (\sigma)$, then we define

$$
T^{f, \theta}: T^{\sigma}(A, P) \rightarrow T^{\tau}(A, Q)
$$

to be the composite $T^{f} \circ T^{\theta}=T^{\theta} \circ T^{f}$. 
3.2.2. For a finite poset $\sigma$ and $p \geq 0$, let $[p]$ be the poset $0<1<2<\cdots<p$ and endow $\sigma \times[p]$ with the lexicographical order. We define

$$
\sigma \star[p]:=\sigma \times[p] \backslash \max \sigma \times\{1, \ldots, p\} .
$$

We denote by $\phi$ (resp. $\varphi$ ) the embedding $\sigma \rightarrow \sigma \times[p]$ (resp. $\sigma \rightarrow \sigma \star[p]$ ), $a \mapsto(a, 0)$. Note that $\varphi^{-1}(\max (\sigma \star[p]))=\max \sigma$ and that $(\sigma \star[p])_{0}=\sigma_{0} \times[p]$.

The following lemma is a variant of Lemma 7.4 in [Su82].

Lemma 3.5. Let $\left\{A_{m}\right\}_{m \in \Xi}$ be a commutative Tor-unital pro ring and $l \geq 0$. Then there exist $p_{l} \geq 0$ and $s_{l}(m) \geq m$ for each $m \in \Xi$ such that:

(i) For all finite posets $\sigma$ and all pseudo-free $\left\{A_{m}\right\}$-modules $\left\{P_{m}\right\}$, the map

$$
\iota_{s_{l}(m), m} H_{l}\left(T^{\varphi}\right): \tilde{H}_{l}\left(T^{\sigma}\left(A_{s_{l}(m)}, P_{s_{l}(m)}\right) \rightarrow \tilde{H}_{l}\left(T^{\sigma \star\left[p_{l}\right]}\left(A_{m}, P_{m}\right)\right)\right.
$$

is equal to zero.

(ii) For all finite posets $\sigma$ and all special morphisms $f:\left\{P_{m}\right\} \rightarrow\left\{Q_{m}\right\}$ between pseudo-free $\left\{A_{m}\right\}$ modules, the map

$$
\iota_{s_{l}(m), m} H_{l}\left(T^{f, \varphi}\right): \tilde{H}_{l}\left(T^{\sigma}\left(A_{s_{l}(m)}, P_{s_{l}(m)}\right) \rightarrow \tilde{H}_{l}\left(T^{\sigma \star\left[p_{l}\right]}\left(A_{m}, Q_{m}\right)\right)\right.
$$

is equal to zero.

Proof. We prove the lemma by induction on $l \geq 0$. The case $l=0$ is clear, here we can take $p_{0}=0$ and $s_{0}(m)=m$. Let $L>0$ and suppose that we have constructed $p_{0} \leq p_{1} \leq \cdots \leq p_{L-1}$ and $s_{0}(m) \leq s_{1}(m) \leq \cdots \leq s_{L-1}(m)$ which satisfy the conditions of the lemma.

Set $q:=p_{L-1}+1$ and $t(m):=s_{L-1}(m)$. First, we prove the following.

Sublemma 3.6. For all finite posets $\sigma$ and all special morphisms $f:\left\{P_{m}\right\} \rightarrow\left\{Q_{m}\right\}$ between pseudofree $\left\{A_{m}\right\}$-modules, the diagram

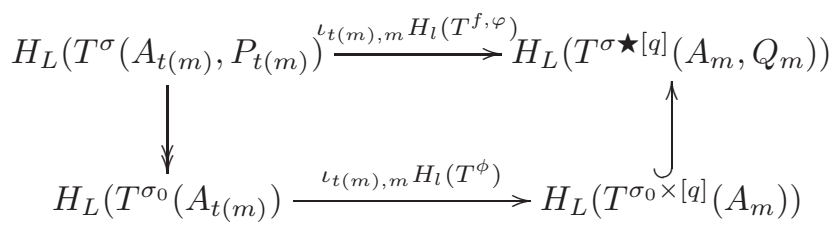

commutes, where the vertical maps are the canonical projection and inclusion.

Proof. Let $f:\left\{P_{m}\right\} \rightarrow\left\{Q_{m}\right\}$ be a special morphism between pseudo-free $\left\{A_{m}\right\}$-modules and $\left\{L_{m}\right\}$ a free basis of $\left\{P_{m}\right\}$ such that $f$ is induced from a map $\left\{L_{m}\right\} \rightarrow\left\{Q_{m}\right\}$. Note that we have an equality $\left\{\underline{\lim }_{i} L_{m}^{(i)}\right\}=\left\{L_{m}\right\}$, where $\left\{L_{m}^{(i)}\right\}$ is a sub-system of $\left\{L_{m}\right\}$ such that each $L_{m}^{(i)}$ is finitely generated and the limit runs around all such systems. Hence, we have

$$
\underset{i}{\underset{i}{l}} C_{*}\left(B M_{n, k}\left(A_{m} \otimes L_{m}^{(i)}\right)\right) \simeq C_{*}\left(B M_{n, k}\left(P_{m}\right)\right)
$$

for every $m$ and $n, k \geq 1$. It follows that

$$
C_{*}\left(B T^{\sigma}\left(A_{m}, P_{m}\right)\right) \simeq \underset{i}{\lim } C_{*}\left(B T^{\sigma}\left(A_{m}, A_{m} \otimes L_{m}^{(i)}\right)\right)
$$

and

$$
H_{*}\left(T^{\sigma}\left(A_{m}, P_{m}\right)\right) \simeq \underset{i}{\lim } H_{*}\left(T^{\sigma}\left(A_{m}, A_{m} \otimes L_{m}^{(i)}\right)\right) .
$$

Consequently, to show the sublemma, we may assume that $\left\{P_{m}\right\}=\left\{A_{m} \otimes_{\mathbb{Z}} L_{m}\right\}$ with $L_{m}$ a free abelian group of finite rank. We may also assume that $\left\{Q_{m}\right\}=\left\{A_{m} \otimes_{\mathbb{Z}} M_{m}\right\}$ with $M_{m}$ a free abelian group of finite rank.

Fix $m \in \Xi$. Let $e_{1}, \ldots, e_{I}$ be a basis of $L_{t(m)}$ and $f_{1}, \ldots, f_{J}$ a basis of $M_{m}$. Since $f$ is special, the map $\iota_{t(m), m} f: P_{t(m)} \rightarrow Q_{m}$ is induced by a map $\alpha: L_{t(m)} \rightarrow Q_{m}$, which sends $e_{i}$ to $\sum_{j} \alpha_{i, j} f_{j}$ with $\alpha_{i, j} \in A_{m}$. We may also denote $\iota_{t(m), m} f$ by $\alpha$. 
If $\alpha=0$, then the diagram

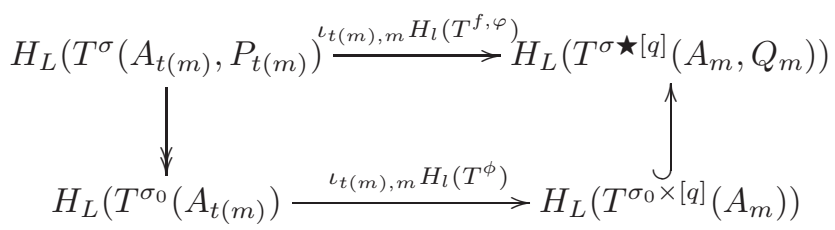

commutes, and thus the sublemma holds in this case. Let $(u, v) \in[1, I] \times[1, J]$ and suppose that the sublemma holds if $\alpha_{i, j}=0$ for $(i, j) \geq(u, v)$ with respect to the lexicographical order. We prove the sublemma in case $\alpha_{i, j}=0$ for $(i, j)>(u, v)$. We define a morphism $\beta: P_{t(m)} \rightarrow Q_{m}$ by sending $e_{i}$ to $\delta_{i, u} f_{v}$.

We define an embedding $\psi: \sigma \rightarrow \sigma \star[q]$ by

$$
\psi(x)= \begin{cases}(x, 0) & \text { if } x \in \max \sigma \\ (x, q) & \text { if } x \notin \max \sigma\end{cases}
$$

Then the image $\tau$ of $\psi$ intersects with $\sigma \star[q-1] \subset \sigma \star[q]$ only at $\max \sigma \times\{0\}$, and thus the composite

$$
T^{\sigma} \stackrel{\text { diag }}{\longrightarrow} T^{\sigma} \times T^{\sigma^{T^{\varphi} \times T^{\psi}}} \longrightarrow T^{\sigma \star[q-1]} \times T^{\tau} \stackrel{\text { prod }}{\longrightarrow} T^{\sigma \star[q]}
$$

is a group homomorphism. Now, we have a group morphism

$$
T^{\sigma}\left(A_{t(m)}, P_{t(m)}\right) \stackrel{\stackrel{T^{\alpha, \varphi}}{\longrightarrow} T^{\sigma \star[q-1]}\left(A_{m}, Q_{m}\right) \longrightarrow}{T^{\beta, \psi}} T^{\tau}\left(A_{m}, Q_{m}\right) \longrightarrow T^{\sigma \star[q]}\left(A_{m}, Q_{m}\right),
$$

which we denote by $T^{\alpha, \varphi} \cdot T^{\beta, \psi}$. Since $q-1=p_{L-1}$ and $t(m)=s_{L-1}(m)$, by the induction hypothesis and by the Künneth formula, we obtain

$$
H_{L}\left(T^{\alpha, \varphi} \cdot T^{\beta, \psi}\right)=H_{L}\left(T^{\alpha, \varphi}\right)+H_{L}\left(T^{\beta, \psi}\right) .
$$

Set

$$
\omega:=\prod_{x \in \sigma_{0}} e_{\varphi(x), \psi(x)}\left(\alpha_{u, v}\right) \in T^{\sigma \star[q]}\left(A_{m}\right) .
$$

We define $\left(\alpha_{i, j}^{\prime}\right) \in M_{I, J}\left(A^{m}\right)$ by $\alpha_{i, j}^{\prime}=\alpha_{i, j}$ unless $(i, j)=(u, v)$ and $\alpha_{u, v}^{\prime}=0$, which induces a map $\alpha^{\prime}: Q_{t(m)} \rightarrow P_{m}$ by sending $e_{i}$ to $\sum_{j} \alpha_{i, j}^{\prime} f_{j}$.

Claim 3.7. We have an equality ${ }^{3}$

$$
\operatorname{Ad}(\omega) \circ\left(T^{\alpha^{\prime}, \varphi} \cdot T^{\beta, \psi}\right)=T^{\alpha, \varphi} \cdot T^{\beta, \psi} .
$$

We calculate the $(k, l)$-entry of (3.2) at $U \in T^{\sigma}\left(A_{t(m)}, P_{t(m)}\right)$. It suffices to do this for;

(1) $(k, l)=(\varphi(x), \varphi(y))$ with $x \in \sigma_{0}$ and $y \in \sigma$.

(2) $(k, l)=(\varphi(x), \psi(y))$ with $x \in \sigma_{0}$ and $y \in \sigma_{0}$.

(3) $(k, l)=(\psi(x), \psi(y))$ with $x \in \sigma_{0}$ and $y \in \sigma_{0}$.

(4) $(k, l)=(\psi(x), \varphi(y))$ with $x \in \sigma_{0}$ and $y \in \sigma$.

\footnotetext{
${ }^{3}$ Here is the only place we need the commutativity of pro rings
} 
Case (1):

$$
\begin{aligned}
& \left(\operatorname{Ad}(\omega) \circ\left(T^{\alpha^{\prime}, \varphi} \cdot T^{\beta, \psi}\right)(U)\right)_{\varphi(x), \varphi(y)} \\
& =\left(\left(T^{\alpha^{\prime}, \varphi} \cdot T^{\beta, \psi}\right)(U)\right)_{\varphi(x), \varphi(y)}+\alpha_{u, v} \cdot\left(\left(T^{\alpha^{\prime}, \varphi} \cdot T^{\beta, \psi}\right)(U)\right)_{\psi(x), \varphi(y)} \\
& =T^{\alpha^{\prime}, \varphi}(U)_{\varphi(x), \varphi(y)}+\alpha_{u, v} \cdot T^{\beta, \psi}(U)_{\psi(x), \varphi(y)} \\
& = \begin{cases}U_{x, y} & \text { if } y \in \sigma_{0} \\
\alpha^{\prime}\left(U_{x, y}\right)+\beta\left(U_{x, y}\right) \alpha_{u, v}=\alpha\left(U_{x, y}\right) & \text { if } y \in \max \sigma\end{cases} \\
& =\left(\left(T^{\alpha, \varphi} \cdot T^{\beta, \psi}\right)(U)\right)_{\varphi(x), \varphi(y)} .
\end{aligned}
$$

Case (2):

$$
\begin{aligned}
& \left(\left(\operatorname{Ad}(\omega) \circ\left(T^{\alpha^{\prime}, \varphi} \cdot T^{\beta, \psi}\right)(U)\right)_{\varphi(x), \psi(y)}\right. \\
& =\alpha_{u, v} \cdot\left(\left(T^{\alpha^{\prime}, \varphi} \cdot T^{\beta, \psi}\right)(U)\right)_{\varphi(x), \varphi(y)}-\left(\left(T^{\alpha^{\prime}, \varphi} \cdot T^{\beta, \psi}\right)(U)\right)_{\psi(x), \psi(y)} \cdot \alpha_{u, v} \\
& =\alpha_{u, v} U_{x, y}-U_{x, y} \alpha_{u, v} \\
& =0 \\
& =\left(\left(T^{\alpha, \varphi} \cdot T^{\beta, \psi}\right)(U)\right)_{\varphi(x), \psi(y)} .
\end{aligned}
$$

Case (3):

$$
\begin{aligned}
& \left(\operatorname{Ad}(\omega) \circ\left(T^{\alpha^{\prime}, \varphi} \cdot T^{\beta, \psi}\right)(U)\right)_{\psi(x), \psi(y)} \\
& =\left(\left(T^{\alpha^{\prime}, \varphi} \cdot T^{\beta, \psi}\right)(U)\right)_{\psi(x), \psi(y)}-\left(\left(T^{\alpha^{\prime}, \varphi} \cdot T^{\beta, \psi}\right)(U)\right)_{\psi(x), \phi(y)} \cdot \alpha_{u, v} \\
& =\left(\left(T^{\alpha, \varphi} \cdot T^{\beta, \psi}\right)(U)\right)_{\psi(x), \psi(y)} .
\end{aligned}
$$

Case (4):

$$
\begin{aligned}
\left(\left(\operatorname{Ad}(\omega) \circ\left(T^{\alpha^{\prime}, \varphi} \cdot T^{\beta, \psi}\right)(U)\right)_{\psi(x), \varphi(y)}\right. & =\left(\left(T^{\alpha^{\prime}, \varphi} \cdot T^{\beta, \psi}(U)\right)_{\psi(x), \varphi(y)}\right. \\
& =\left(\left(T^{\alpha^{\prime}, \varphi} \cdot T^{\beta, \psi}(U)\right)_{\psi(x), \varphi(y)} .\right.
\end{aligned}
$$

Consequently, we obtain the equality in the claim.

Again, by the induction hypothesis and by the Künneth formula, we obtain

$$
\begin{aligned}
H_{L}\left(T^{\alpha, \varphi} \cdot T^{\beta, \psi}\right) & =H_{L}\left(T^{\alpha^{\prime}, \varphi} \cdot T^{\beta, \psi}\right) \\
& =H_{L}\left(T^{\alpha^{\prime}, \varphi}\right)+H_{L}\left(T^{\beta, \psi}\right) .
\end{aligned}
$$

It follows from $(3.1,3.2)$ that

$$
H_{L}\left(T^{\alpha, \varphi}\right)=H_{L}\left(T^{\alpha^{\prime}, \varphi}\right) .
$$

Therefore, by induction, we get the sublemma.

We return to the proof of Lemma 3.5. We prove $(i)_{l=L}$. Let $\left\{P_{m}\right\}$ be a pseudo-free $\left\{A_{m}\right\}$-module. Let $\left\{P_{m}[-]\right\}$ be a pro resolution of $\left\{P_{m}\right\}$, cf. Lemma 2.6. Then, by Proposition 2.4 and Corollary 3.4, $\left\{P_{m}[-\geq 0]\right\} \rightarrow\left\{P_{m}\right\}$ induces a pro isomorphism

$$
\Theta:\left\{H_{L}\left(T^{\sigma}\left(A_{m}, P_{m}[-\geq 0]\right)\right)\right\}_{m} \stackrel{\sim}{\rightarrow}\left\{H_{L}\left(T^{\sigma}\left(A_{m}, P_{m}\right)\right)\right\}_{m} .
$$

In fact, for each $m \in \Xi$ there exists $r(m) \geq m$, which does not depend on $\left\{P_{m}\right\},\left\{P_{m}[-]\right\}$ and $\sigma$, such that the maps $\iota_{r(m), m}: \operatorname{ker} \Theta_{r(m)} \rightarrow \operatorname{ker} \Theta_{m}$ and $\iota_{r(m), m}: \operatorname{coker} \Theta_{r(m)} \rightarrow$ coker $\Theta_{m}$ are equal to zero, cf. Proposition 2.5.

We set

$$
\begin{gathered}
p:=p_{L}:=\left(\prod_{l=1}^{L-1}\left(p_{l}+1\right)\right)(q+1)-1, \\
s(m):=s_{L}(m):=r\left(s_{1}\left(\cdots\left(s_{L-1}(t(m))\right) \cdots\right)\right) .
\end{gathered}
$$


We claim that $(i)_{l=L}$ holds with these definitions. We prove it by induction on $n:=\# \sigma \geq 1$. The case $n=1$ is clear, and so let $n>1$.

By Lemma 3.3, we have

$$
C_{*}\left(B T^{\sigma}\left(A_{m}, P_{m}\left[-_{\geq 0}\right]\right)=C_{*}\left(E T^{\sigma_{0}}\left(A_{m}\right)\right) \otimes_{T^{\sigma_{0}\left(A_{m}\right)}} C_{*}\left(B M_{\sigma_{0}, \max \sigma}\left(P_{m}\left[-_{\geq 0}\right]\right)\right)\right.
$$

and thus we have a first quadrant spectral sequence

$$
\left(E_{s, t}^{1}\right)_{m}^{\sigma}=H_{t}\left(T^{\sigma}\left(A_{m}, P_{m}[s]\right)\right) \Rightarrow H_{s+t}\left(T^{\sigma}\left(A_{m}, P_{m}[-\geq 0]\right)\right) .
$$

It is clear that $\left(E_{s, 0}^{2}\right)_{m}^{\sigma}=0$ for $s>0$. Hence, the spectral sequence induces a filtration

$$
0=F_{-1, m}^{\sigma} \subset F_{0, m}^{\sigma} \subset \cdots \subset F_{L-1, m}^{\sigma}=H_{L}\left(T^{\sigma}\left(A_{m}, P_{m}[-\geq 0]\right)\right)
$$

with $F_{i, m}^{\sigma} / F_{i-1, m}^{\sigma}$ a subquotient of $H_{L-i}\left(T^{\sigma}\left(A_{m}, P_{m}[i]\right)\right)$.

Note that the map $\varphi: \sigma \rightarrow \sigma \star[p]$ induces a morphism of spectral sequences

$$
\begin{gathered}
\left(E_{s, t}^{1}\right)_{m}^{\sigma}=H_{t}\left(T^{\sigma}\left(A_{m}, P_{m}[s]\right)\right) \Longrightarrow \\
\downarrow \\
\left(E_{s, t}^{1}\right)_{m}^{\sigma \star[p]}=H_{t}\left(T^{\sigma \star[p]}\left(A_{m}, P_{m}[s]\right)\right) \Longrightarrow H_{s+t}\left(T^{\sigma \star[p]}\left(A_{m}, P_{m}\left[A_{m}, P_{m}[-\geq 0]\right)\right) .\right.
\end{gathered}
$$

By the induction hypothesis, the induced map

$$
F_{i, s_{L-i}(m)}^{\sigma} / F_{i-1, s_{L-i}(m)}^{\sigma} \rightarrow F_{i, m}^{\sigma \star\left[p_{L-i}\right]} / F_{i-1, m}^{\sigma \star\left[p_{L-i}\right]}
$$

is zero for $1 \leq i \leq L-1$. Also, observe that $(\sigma \star[a]) \star[b]=\sigma \star[(a+1)(b+1)-1]$. It follows that, by putting $s^{\prime}(m):=s_{1}\left(\cdots\left(s_{L-1}(t(m))\right) \cdots\right)$ and $p^{\prime}:=\prod_{l=1}^{L-1}\left(p_{l}+1\right)-1$, the canonical map

$$
\iota_{s^{\prime}(m), t(m)} H_{l}\left(T^{\varphi}\right): H_{L}\left(T^{\sigma}\left(A_{s^{\prime}(m)}, P_{s^{\prime}(m)}\left[-_{\geq 0}\right]\right)\right) \rightarrow H_{L}\left(T^{\sigma \star\left[p^{\prime}\right]}\left(A_{t(m)}, P_{t(m)}\left[-_{\geq 0}\right]\right)\right)
$$

factors through $F_{0, t(m)}^{\sigma \star\left[p^{\prime}\right]}$

Now, we have lifts in the commutative diagram

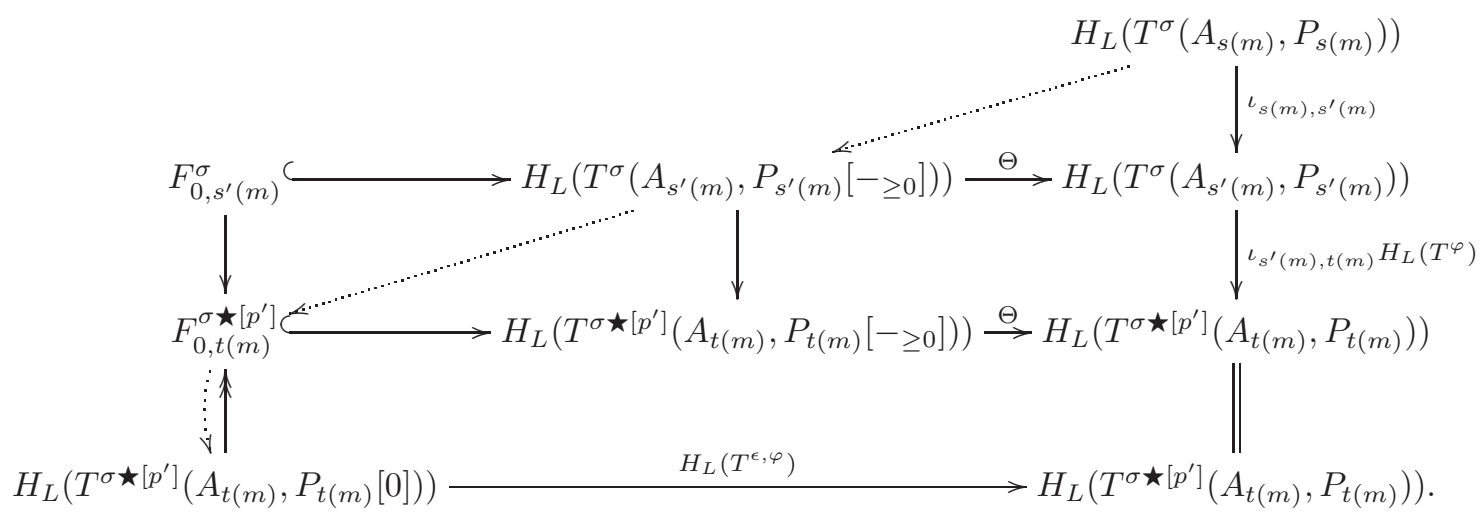

Consider the following diagram

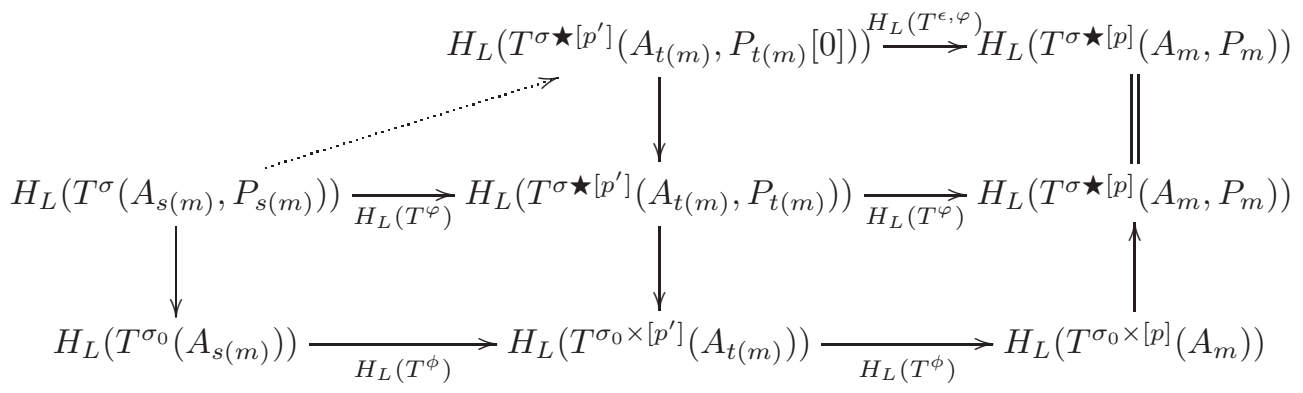


where we omit the structured maps $\iota_{*, *}$. The right rectangle commutes by Sublemma 3.6, though the lower right square may not commute. It follows from a simple diagram chase that the bottom rectangle commutes. Therefore, by the induction hypothesis for $n=\# \sigma$, the middle composite $\iota_{s(m), m} H_{L}\left(T^{\varphi}\right)$ equals zero.

Finally, $(i i)_{l=L}$ follows immediately from $(i)_{l=L}$ and Sublemma 3.6.

3.2.3. Let $\sigma$ be a partial ordering on $\{1, \ldots, n\}$. Then we can naturally regard $T^{\sigma}(A)$ as a subgroup of $\mathrm{GL}_{n}(A)$. For $k \geq 0$, we define ${ }^{k} \tilde{\sigma}$ to be the partial ordering on $\{1, \ldots, n+k\}$ obtained from $\sigma$ by adding the relations $i<n+j$ for $i \in\{1, \ldots, n\}$ and $1 \leq j \leq k$. Set

$$
{ }^{k} \tilde{T}^{\sigma}(A, P):=\left\{\begin{array}{ll}
T^{\sigma}(A) & \text { if } k=0 \\
T^{k} \tilde{\sigma}(A, P) & \text { if } k \geq 1
\end{array}=T^{\sigma}(A) \ltimes M_{n, k}(P) .\right.
$$

We write $\Pi_{n}$ for the set of all partial orderings on $\{1, \ldots, n\}$.

Corollary 3.8. Let $\mathbf{A}$ be a commutative Tor-unital pro ring and $l, n \geq 0$. Let $\sigma_{1}, \ldots, \sigma_{t} \in \Pi_{n}$. Then there exists $p \geq 0$ such that the canonical map

$$
\tilde{H}_{l}\left(\bigcup_{i=1}^{t} B^{k} \tilde{T}^{\sigma_{i}}(\mathbf{A}, \mathbf{P})\right) \rightarrow \tilde{H}_{l}\left(\bigcup_{i=1}^{t} B^{k} \tilde{T}^{\sigma_{i} \times[p]}(\mathbf{A}, \mathbf{P})\right)
$$

is equal to zero as a pro morphism for all $k \geq 0$ and all pseudo-free $\mathbf{A}$-modules $\mathbf{P}$, where the unions are taken in $B\left(\mathrm{GL}_{n}(\mathbf{A}) \ltimes M_{n, k}(\mathbf{P})\right)$ and $B\left(\mathrm{GL}_{n(p+1)}(\mathbf{A}) \ltimes M_{n(p+1), k}(\mathbf{P})\right)$ respectively.

In particular, there exists $N \geq n$ such that the canonical map

$$
\tilde{H}_{l}\left(\bigcup_{\sigma \in \Pi_{n}} B^{k} \tilde{T}^{\sigma}(\mathbf{A}, \mathbf{P})\right) \rightarrow \tilde{H}_{l}\left(\bigcup_{\sigma \in \Pi_{N}} B^{k} \tilde{T}^{\sigma}(\mathbf{A}, \mathbf{P})\right)
$$

is equal to zero for all $k \geq 0$ and all pseudo-free $\mathbf{A}$-modules $\mathbf{P}$.

Proof. Note that

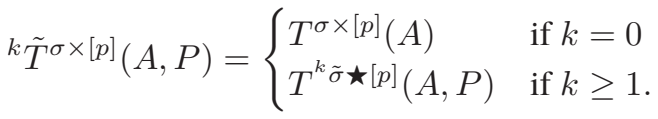

Hence, the case $t=1$ is true by Lemma 3.5. Let $t>1$ and suppose that the corollary holds for $s<t$.

We abbreviate ${ }^{k} \tilde{T}^{\sigma}(\mathbf{A}, \mathbf{P})$ as $\tilde{T}^{\sigma}$. Set $\sigma_{i, t}:=\sigma_{i} \cap \sigma_{t}$. Then we have a commutative diagram

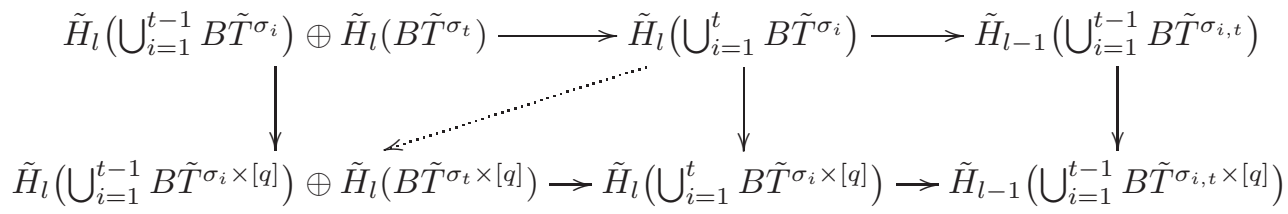

with exact rows. By the induction hypothesis, the right vertical map is zero for some $q \geq 0$. Thus, there exists a lift as indicated above. Again, by the induction hypothesis, there exists $q^{\prime} \geq 0$ such that the map

$$
\tilde{H}_{l}\left(\bigcup_{i=1}^{t-1} B \tilde{T}^{\sigma_{i}}\right) \oplus \tilde{H}_{l}\left(B \tilde{T}^{\sigma_{t}}\right) \rightarrow \tilde{H}_{l}\left(\bigcup_{i=1}^{t-1} B \tilde{T}^{\sigma_{i} \times\left[q^{\prime}\right]}\right) \oplus \tilde{H}_{l}\left(B \tilde{T}^{\sigma_{t} \times\left[q^{\prime}\right]}\right)
$$

is zero. It follows from $\left(\sigma_{i} \times[q]\right) \times\left[q^{\prime}\right]=\sigma_{i} \times\left[q^{\prime \prime}\right], q^{\prime \prime}:=(q+1)\left(q^{\prime}+1\right)-1$, that the map

$$
\tilde{H}_{l}\left(\bigcup_{i=1}^{t} B \tilde{T}^{\sigma_{i}}\right) \rightarrow \tilde{H}_{l}\left(\bigcup_{i=1}^{t} B \tilde{T}^{\sigma_{i} \times\left[q^{\prime \prime}\right]}\right)
$$

is zero. This completes the proof. 
3.3. The pro acyclicity theorem. Recall that ${ }^{k} \tilde{T}^{\sigma}(A)=T^{\sigma}(A) \ltimes M_{n, k}(A)$ for $\sigma \in \Pi_{n}$.

Theorem 3.9. Let $\mathbf{A}$ be a commutative Tor-unital pro ring and $l \geq 0$. Then:

(i) For $n \geq 2 l+1$ and for any $k \geq 0$,

$$
\tilde{H}_{l}\left(\bigcup_{\sigma \in \Pi_{n}} B^{k} \tilde{T}^{\sigma}(\mathbf{A})\right)=0
$$

where the union is taken in $B\left(\mathrm{GL}_{n}(\mathbf{A}) \ltimes M_{n, k}(\mathbf{A})\right)$.

(ii) For $n \geq 2 l$ and for any $k \geq 0$, the canonical map

$$
H_{l}\left(\bigcup_{\sigma \in \Pi_{n}} B T^{\sigma}(\mathbf{A})\right) \rightarrow H_{l}\left(\bigcup_{\sigma \in \Pi_{n}} B^{k} \tilde{T}^{\sigma}(\mathbf{A})\right)
$$

is a pro isomorphism.

Proof. We write ${ }^{k} \tilde{X}_{n}(\mathbf{A})=\bigcup_{\sigma \in \Pi_{n}} B^{k} \tilde{T}^{\sigma}(\mathbf{A})$ and $X_{n}(\mathbf{A})={ }^{0} \tilde{X}_{n}(\mathbf{A})$.

We prove the theorem by induction on $l$. The case $l=0$ is trivial. Let $L>0$ and suppose that the theorem holds for $l<L$.

Sublemma 3.10. Let $k \geq 0$. The canonical map

$$
H_{L}\left({ }^{k} \tilde{X}_{n}(\mathbf{A})\right) \rightarrow H_{L}\left({ }^{k} \tilde{X}_{n+1}(\mathbf{A})\right)
$$

is a pro epimorphism for $n \geq 2 L$ and a pro isomorphism for $n \geq 2 L+1$.

Proof. Let us introduce some notation. Let $A$ be a ring, $\sigma \in \Pi_{n}$ and $1 \leq i \leq n$. We define $T_{n}^{\sigma, i}(A)$ be the subgroup of $T_{n}^{\sigma}(A)$ consisting of all $\alpha$ with $\alpha_{i, j}=\alpha_{j, i}=0$ for $i \neq j$. For $k \geq 0$, we set

$$
{ }^{k} \tilde{X}_{n}^{i}(A):=\bigcup_{\sigma \in \Pi_{n}} B T^{k} \tilde{\sigma}, i(A)
$$

and write ${ }^{k} \tilde{X}_{n}^{i_{1}, \ldots, i_{p}}(A)$ for the intersection of ${ }^{k} \tilde{X}_{n}^{i_{1}}(A), \ldots,{ }^{k} \tilde{X}_{n}^{i_{p}}(A)$. Then it is easy to see that ${ }^{k} \tilde{X}_{n}^{i_{1}, \ldots, i_{p}}(A) \simeq$ ${ }^{k} \tilde{X}_{n-p}(A)$.

Consider the spectral sequence

$$
{ }^{k} \tilde{E}_{p, q}^{1}=\bigsqcup_{i_{0}, \ldots, i_{p}} H_{q}\left({ }^{k} \tilde{X}_{n+1}^{i_{0}, \ldots, i_{p}}(\mathbf{A})\right) \Rightarrow H_{p+q}\left(\bigcup_{1 \leq i \leq n+1}{ }^{k} \tilde{X}_{n+1}^{i}(\mathbf{A})\right) .
$$

Since ${ }^{k} \tilde{X}_{n+1}^{i_{0}, \ldots, i_{p}}(\mathbf{A}) \simeq{ }^{k} \tilde{X}_{n-p}(\mathbf{A})$, it follows form the induction hypothesis that

$$
{ }^{k} \tilde{E}_{0, L}^{2} \simeq H_{L}\left({ }^{k} \tilde{X}_{n}(\mathbf{A})\right)
$$

for $n \geq 2 L$. Hence, the canonical map

$$
H_{L}\left({ }^{k} \tilde{X}_{n}(\mathbf{A})\right) \rightarrow H_{L}\left(\bigcup_{1 \leq i \leq n+1}{ }^{k} \tilde{X}_{n+1}^{i}(\mathbf{A})\right)
$$

is a pro epimorphism for $n \geq 2 L$ and a pro isomorphism for $n \geq 2 L+1$. According to [Su82, Corollary 6.6, see also the remark before Theorem 7.1 $]^{4}$, the canonical map

$$
H_{L}\left(\bigcup_{1 \leq i \leq n+1}{ }^{k} \tilde{X}_{n+1}^{i}(\mathbf{A})\right) \rightarrow H_{L}\left({ }^{k} \tilde{X}_{n+1}(\mathbf{A})\right)
$$

is a levelwise surjection for $n \geq 2 L$ and a levelwise bijection for $n \geq 2 L+1$. Bringing these together, we obtain the sublemma.

\footnotetext{
${ }^{4}$ The proof works for non-unital rings as it is.
} 
We show $(i)_{l=L}$. Suppose that $n \geq 2 L+1$. According to Corollary 3.8, the canonical map

$$
H_{L}\left({ }^{k} \tilde{X}_{n}(\mathbf{A})\right) \rightarrow H_{L}\left({ }^{k} \tilde{X}_{N}(\mathbf{A})\right)
$$

is zero for some $N \geq n$. On the other hand, by Sublemma 3.10, this map is a pro isomorphism, and thus $H_{L}\left({ }^{k} \tilde{X}_{n}(\mathbf{A})\right)=0$.

To get $(i i)_{l=L}$, it remains to show that the canonical map

$$
H_{L}\left(X_{2 L}(\mathbf{A})\right) \rightarrow H_{L}\left({ }^{k} \tilde{X}_{2 L}(\mathbf{A})\right)
$$

is a pro isomorphism. By the spectral sequences (3.3), we have a commutative diagram

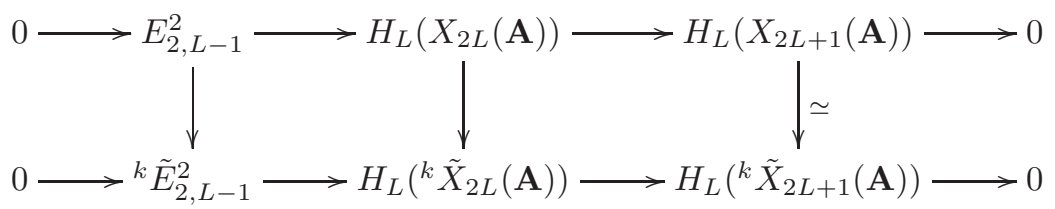

with exact rows. Hence, it is enough to show that $E_{2, L-1}^{2} \rightarrow{ }^{k} \tilde{E}_{2, L-1}^{2}$ is a pro isomorphism; equivalently it is a pro epimorphism. This follows from the diagram

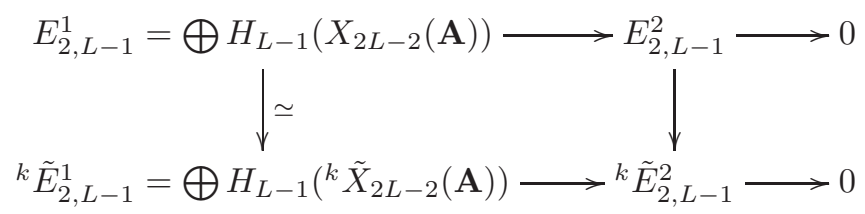

with exact rows. 


\section{Homology PRo STABility}

In this section, we completes the proof of Theorem 0.3. We follow Suslin [Su96], generalizing his argument to the pro setting.

4.1. Volodin spaces. Let $G$ be a group and $\left\{G_{i}\right\}_{i \in I}$ a family of subgroups of $G$. We define the Volodin space $V\left(G,\left\{G_{i}\right\}_{i \in I}\right)$ to be the simplicial subset of $E G$ formed by simplices $\left(g_{0}, \ldots, g_{p}\right)$ such that there exists $i \in I$ and $g_{j} g_{k}^{-1} \in G_{i}$ for all $0 \leq j, k \leq p$.

The simplicial subset $V\left(G,\left\{G_{i}\right\}_{i \in I}\right) \subset E G$ is stable under the right action of $G$, and $V\left(G,\left\{G_{i}\right\}\right) / G=$ $\bigcup_{i \in I} B G_{i}$. Hence, we have a spectral sequence

$$
E_{p, q}^{2}=H_{p}\left(G, H_{q}\left(V\left(G,\left\{G_{i}\right\}_{i \in I}\right)\right)\right) \Rightarrow H_{p+q}\left(\bigcup_{i \in I} B G_{i}\right) .
$$

Let $A$ be a ring. We consider the Volodin space

$$
V_{n}(A):=V\left(E_{n}(A),\left\{T^{\sigma}(A)\right\}_{\sigma \in \Pi_{n}}\right) .
$$

The permutation group $\Sigma_{n}$ acts on $V_{n}(A)$ by conjugation, and $E_{n}(A)$ acts on $V_{n}(A)$ by the right multiplication.

Here are some properties of the Volodin spaces we need.

Lemma 4.1 (Suslin-Wodzicki [SW92, Corollary 2.7]). Let $n \geq 1$ and $k \geq 0$. The canonical projection and the inclusion

$$
V_{n}(A) \rightleftarrows V\left(\left(\begin{array}{cc}
E_{n}(A) & * \\
0 & 1_{k}
\end{array}\right),\left\{\left(\begin{array}{cc}
T^{\sigma}(A) & * \\
0 & 1_{k}
\end{array}\right)\right\}_{\sigma \in \Pi_{n}}\right)
$$

are mutually inverse homotopy equivalences.

Lemma 4.2 (Suslin-Wodzick [SW92, Lemma 2.8]). For every $n, l \geq 0$, the action of $E_{n+1}\left(A^{2}\right)$ on the image of the canonical map

is trivial.

$$
H_{l}\left(V_{n}(A)\right) \rightarrow H_{l}\left(V_{n+1}(A)\right)
$$

Corollary 4.3. Let $\mathbf{A}$ be a pro ring such that $\mathbf{A} / \mathbf{A}^{2}=0$. Then, for every $n, l \geq 0$, the action of $E_{n+1}(\mathbf{A})$ on the image of the canonical map

$$
H_{l}\left(V_{n}(\mathbf{A})\right) \rightarrow H_{l}\left(V_{n+1}(\mathbf{A})\right)
$$

is pro trivial.

Proof. Write $\mathbf{A}=\left\{A_{m}\right\}$. By the assumption, there exists $s \geq m$ for each $m$ such that $\iota_{s, m} A_{s} \subset A_{m}^{2}$. Hence, given $x$ in the image of $H_{l}\left(V_{n}\left(A_{s}\right)\right) \rightarrow H_{l}\left(V_{n+1}\left(A_{s}\right)\right)$ and $g \in E_{n}\left(A_{s}\right)$, we have $\iota_{s, m}(g x)=$ $\iota_{s, m}(x)$.

4.2. Van der Kallen's acyclicity. Let $A$ be a ring and $n \geq 1$. Fix a unital $\operatorname{ring} R$ which contains $A$ as a two sided ideal. Let $I$ be a finite subset of $\{1, \ldots, n\}$ and $R^{n}$ the free right $R$-module with basis $e_{1}, \ldots, e_{n}$. A map $f: I \rightarrow R^{n}$ is called an A-unimodular function if $\{f(i)\}_{i \in I}$ forms a basis of a free direct summand of $R^{n}$ and $f(i) \equiv e_{i}$ modulo $A$. We denote by Uni ${ }_{A, n}=\operatorname{Uni}_{A, n}(R)$ the set of all $A$-unimodular functions $f: I \rightarrow R^{n}$, which does not depend on $R$.

We define the associated semi-simplicial set as follows: A $p$-simplex is an $A$-unimodular function $f \in \operatorname{Uni}_{A, n}$ with $|\operatorname{dom} f|=p+1$. The $i$-th face $d_{i}:\left(\operatorname{Uni}_{A, n}\right)_{p} \rightarrow\left(\operatorname{Uni}_{A, n}\right)_{p-1}, 0 \leq i \leq p$, is defined by

$$
\left.\left(f, \operatorname{dom} f=\left\{i_{0}, \ldots, i_{p}\right\}\right) \mapsto f\right|_{\left\{i_{0}, \ldots, \hat{i}_{k}, \ldots, i_{p}\right\}} .
$$

As in the preceding section, for a semi-simplicial set $X$, we denote by $C_{*}(X)$ the complex freely generated by $X_{*}$ with the differential being the alternating sum of the faces.

The following result is proved by van der Kallen [vdK80] in case $A$ is unital, and the proof can be easily modified for non-unital rings. We can also find the complete proof in [Su96, §2].

Theorem 4.4. $\tilde{H}_{l}\left(C_{*}\left(\mathrm{Uni}_{A, n}\right)\right)=0$ for $n \geq l+\operatorname{sr}(A)+1$. 
Let $\mathrm{SUni}_{A, n}$ (resp. $\overline{\mathrm{SUni}}_{A, n}(R)$ ) be the set of all unimodular functions $f \in \operatorname{Uni}_{A, n}(R)$ for which there exists $\alpha \in E_{n}(A)$ (resp. $\alpha \in E_{n}(R, A)$ ) such that $f(i)=e_{i} \alpha$ for all $i \in \operatorname{dom} f$. Then SUni ${ }_{A, n}$ and $\overline{\mathrm{SUni}}_{A, n}(R)$ are sub semi-simplicial sets of $\mathrm{Uni}_{A, n}(R)$.

\section{Corollary 4.5.}

(i) $\tilde{H}_{l}\left(C_{*}\left(\overline{\operatorname{SUni}}_{A, n}(R)\right)\right)=0$ for $n \geq l+\operatorname{sr}(A)+1$.

(ii) Let $\mathbf{A}$ be a pro ring such that $\mathbf{A} / \mathbf{A}^{2}=0$. Then

$$
\tilde{H}_{l}\left(C_{*}\left(\mathrm{SUni}_{\mathbf{A}, n}\right)\right)=0
$$

as pro abelian groups for $n \geq l+\operatorname{sr}(\mathbf{A})+1$.

Proof. (i) See [Su96, Corollary 2.8].

(ii) Let $\mathbf{R}$ be a unital pro ring which contains $\mathbf{A}$ as a two-sided ideal. By Corollary 1.3, the canonical map SUni $\mathbf{A}, n \rightarrow \overline{\operatorname{SUni}}_{\mathbf{A}, n}(\mathbf{R})$ is a pro isomorphism. Hence, (ii) follows from (i).

4.3. Homology pro stability for $V_{n}$ and $E_{n}$. We say that a levelwise action of a pro group $\left\{G_{m}\right\}$ on a pro object $\left\{M_{m}\right\}$ is pro trivial if there exists $s \geq m$ for each $m$ such that $\iota_{s, m}(g x)=\iota_{s, m}(x)$ for all $g \in G_{s}$ and $x \in M_{s}$.

Theorem 4.6. Let $\mathbf{A}$ be a commutative Tor-unital pro ring. Let $r=\max (\operatorname{sr}(\mathbf{A}), 2)$ and $l \geq 0$. Then:

(i) The canonical map

$$
H_{l}\left(V_{n}(\mathbf{A})\right) \rightarrow H_{l}\left(V_{n+1}(\mathbf{A})\right)
$$

is a pro epimorphism for $n \geq 2 l+r+1$ and a pro isomorphism for $n \geq 2 l+r+2$.

(ii) The conjugate action of $\Sigma_{n}$ on $H_{l}\left(V_{n}(\mathbf{A})\right)$ is pro trivial for $n \geq 2 l+r+2$.

(iii) The action of $E_{n}(\mathbf{A})$ on $H_{l}\left(V_{n}(\mathbf{A})\right)$ is pro trivial for $n \geq 2 l+r+2$.

(iv) The canonical map

$$
H_{l}\left(E_{n}(\mathbf{A})\right) \rightarrow H_{l}\left(\left(\begin{array}{cc}
E_{n}(\mathbf{A}) & * \\
0 & 1_{k}
\end{array}\right)\right)
$$

is a pro isomorphism for $n \geq 2 l+r-2$ and for any $k \geq 0$.

(v) The conjugate action of $\Sigma_{n}$ on $H_{l}\left(E_{n}(\mathbf{A})\right)$ is pro trivial for $n \geq 2 l+r-1$.

(vi) The canonical map

$$
H_{l}\left(E_{n}(\mathbf{A})\right) \rightarrow H_{l}\left(E_{n+1}(\mathbf{A})\right)
$$

is a pro epimorphism for $n \geq 2 l+r-2$ and a pro isomorphism for $n \geq 2 l+r-1$.

We prove Theorem 4.6 by induction on $l$. The case $l=0$ is clear. Also, $(i v, v, v i)_{l=1}$ holds for the obvious reasons: $(v, v i)_{l=1}$ follows from that $H_{1}\left(E_{n}(\mathbf{A})\right)=0$ for $n \geq 3$. For $(v i)_{l=1}$, note that we have a levelwise exact sequence

$$
M_{n, k}(A)_{E_{n}(\mathbf{A})} \longrightarrow H_{1}\left(E_{n}(\mathbf{A}) \ltimes M_{n, k}(\mathbf{A})\right) \longrightarrow H_{1}\left(E_{n}(\mathbf{A})\right) \longrightarrow 0,
$$

and it is easy to see that $M_{n, k}(\mathbf{A})_{E_{n}(\mathbf{A})}=0$ for $n \geq 2$.

Let $L>0$. The proof is divided into the four steps.

Step 1: $(i, i i, i i i)_{l<L-1} \Rightarrow(i i i)_{l=L-1}$.

Step 2: $(i i i)_{l \leq L-1},(i v)_{l<L+1} \Rightarrow(i v)_{l=L+1}$.

Step 3: $(i v)_{l \leq L+1},(v, v i)_{l<L+1} \Rightarrow(v, v i)_{l=L+1}$.

Step 4: $(i, i i)_{l<L-1},(i i i)_{l \leq L-1},(v i)_{l \leq L+1} \Rightarrow(i, i i)_{l=L-1}$.

4.4. Step 1: Covering argument I. Suppose that $(i, i i, i i i)_{l<L-1}$ hold. We show $(i i i)_{l=L-1} .{ }^{5}$

\footnotetext{
${ }^{5}$ In this step, we only need $\operatorname{Tor}_{1}^{\mathbb{Z} \ltimes \mathbf{A}}(\mathbb{Z}, \mathbb{Z})=\mathbf{A} / \mathbf{A}^{2}=0$.
} 
4.4.1. Covering spectral sequence. Let $A$ be a ring. For $I \subset\{1, \ldots, n\}$, let $\Pi_{n}^{I}$ be the set of all partial ordering $\sigma$ of $\{1, \ldots, n\}$ for which every $i \in I$ is maximal. Set $V_{n}(A)^{I}:=V_{n}\left(E_{n}(A),\left\{T^{\sigma}(A)\right\}_{\sigma \in \Pi_{n}^{I}}\right)$. Then $V_{n}(A)=\bigcup_{i=1}^{n} V_{n}(A)^{i}$, and there is a spectral sequence

$$
E_{p, q}^{1}(A)=\bigsqcup_{|I|=p+1} H_{q}\left(V_{n}(A)^{I}\right) \Rightarrow H_{p+q}\left(V_{n}(A)\right) .
$$

Let SUni ${ }_{A, n}^{I}$ be the subset of SUni ${ }_{A, n}$ consisting of those functions $f$ with $\operatorname{dom} f=I$. We define a $\operatorname{map} \phi: V_{n}(A)^{I} \rightarrow \mathrm{SUni}_{A, n}^{I}$ by $\phi\left(\alpha_{0}, \ldots, \alpha_{q}\right)(i)=e_{i} \alpha_{0}, i \in I$. Then $\phi$ is a morphism of simplicial sets regarding $\mathrm{SUni}_{A, n}^{I}$ as a constant simplicial set, and the inverse image of the unimodular function $f_{0}: i \mapsto$ $e_{i}$ is $V\left(E_{n}(A)^{I},\left\{T^{\sigma}(A)\right\}_{\sigma \in \Pi_{n}^{I}}\right)$, where $E_{n}(A)^{I}$ is the subgroup of $E_{n}(A)$ generated by elementary matrices $\alpha$ such that $e_{i} \alpha=e_{i}$ for all $i \in I$. For each $f \in \operatorname{SUni}_{A, n}^{I}$, choose $\Lambda(f) \in E_{n}(A)$ with $f(i)=$ $e_{i} \Lambda(f), i \in I$. Since the map $\phi$ is $E_{n}(A)$-equivariant, $\Lambda(f)$ gives an isomorphism $\phi^{-1}\left(f_{0}\right) \simeq \phi^{-1}(f)$ and

$$
\mathrm{SUni}_{A, n}^{I} \times V\left(E_{n}(A)^{I},\left\{T^{\sigma}(A)\right\}_{\sigma \in \Pi_{n}^{I}}\right) \stackrel{\sim}{\rightarrow} V_{n}(A)^{I}, \quad(f, u) \mapsto u \Lambda(f) .
$$

Also, the conjugation by the shuffle permutation $\sigma_{I}, \sigma_{I}\{n-p, \ldots, n\}=I$, gives an isomorphism

$$
V\left(E_{n}(A)^{n-p, \ldots, n},\left\{T^{\sigma}(A)\right\}_{\sigma \in \Pi_{n}^{n-p, \ldots, n}}\right) \stackrel{\sim}{\longrightarrow} V\left(E_{n}(A)^{I},\left\{T^{\sigma}(A)\right\}_{\sigma \in \Pi_{n}^{I}}\right) .
$$

Hence, we get an isomorphism

$$
\Phi_{\Lambda}: C_{p}\left(\mathrm{SUni}_{A, n}\right) \otimes H_{q}\left(V\left(E_{n}(A)^{n-p, \ldots, n},\left\{T^{\sigma}(A)\right\}_{\sigma \in \Pi_{n}^{n-p, \ldots, n}}\right)\right) \stackrel{\sim}{\rightarrow} E_{p, q}^{1}(A) .
$$

For another choice of $\Lambda^{\prime}$, there exists $\left\{\gamma(f) \in E_{n}(A)^{n-p, \ldots, n}\right\}_{f \in \operatorname{SUni}_{A, n}}$ such that $\Phi_{\Lambda^{\prime}}(f, u)=\Phi_{\Lambda}(f, u \gamma(f))$.

Under the isomorphism $\Phi_{\Lambda}$, the differential $d^{1}: E_{p, q} \rightarrow E_{p-1, q}$ is given by, for $f \in \mathrm{SUni}_{A, n}^{I}$ and $u \in H_{q}\left(V\left(E_{n}(A)^{n-p, \ldots, n},\left\{T^{\sigma}(A)\right\}_{\sigma \in \Pi_{n}^{n-p, \ldots, n}}\right)\right.$,

$$
d^{1}(f \otimes u)=\sum_{k=0}^{p}(-1)^{k} d_{k} f \otimes \tau_{I, k}(\delta u) \tau_{I, k}^{-1} \alpha_{k} .
$$

Here, $\alpha_{k}$ is a certain element in $E_{n}(A)^{n-p+1, \ldots, n}, \tau_{I, k}:=\sigma_{I \backslash\left\{i_{k}\right\}}^{-1} \sigma_{I}$, and $\delta$ is the map induced from the canonical embedding $E_{n}(A)^{n-p, \ldots, n} \rightarrow E_{n}(A)^{n-p+1, \ldots, n}$.

4.4.2. Pro arguments. We write $\mathbf{A}=\left\{A_{m}\right\}_{m \in \Xi}$.

Set $\bar{E}_{n}(\mathbf{A}):=\mathrm{GL}_{n}(\mathbf{A}) \cap E(\mathbf{A})$. Then the canonical maps

$$
\begin{aligned}
& H_{q}\left(V\left(\bar{E}_{n-p-1}(\mathbf{A}),\left\{T^{\sigma}(\mathbf{A})\right\}_{\sigma \in \Pi_{n-p-1}}\right)\right) \\
& \downarrow \simeq \\
& H_{q}\left(V\left(\bar{E}_{n}(\mathbf{A})^{n-p, \ldots, n},\left\{T^{\sigma}(\mathbf{A})\right\}_{\sigma \in \Pi_{n}^{n-p, \ldots, n}}\right)\right) \\
& \downarrow \simeq \\
& H_{q}\left(V\left(\left(\begin{array}{cc}
\bar{E}_{n-p-1}(\mathbf{A}) & * \\
0 & 1_{p+1}
\end{array}\right),\left\{\left(\begin{array}{cc}
T^{\sigma}(\mathbf{A}) & * \\
0 & 1_{p+1}
\end{array}\right)\right\}_{\sigma \in \Pi_{n-p-1}}\right)\right)
\end{aligned}
$$

are levelwise isomorphisms. Indeed, the second map is an isomorphism by definition and the composite is an isomorphism by Lemma 4.1. Hence, by Theorem 1.6, the canonical map

$$
\lambda: H_{q}\left(V_{n-p-1}(\mathbf{A})\right) \rightarrow H_{q}\left(V\left(E_{n}(\mathbf{A})^{n-p, \ldots, n},\left\{T^{\sigma}(\mathbf{A})\right\}_{\sigma \in \Pi_{n}^{n-p, \ldots, n}}\right)\right)
$$

is a pro isomorphism for $n-p-1 \geq r+1$.

Suppose that $q<L-1$ and $n-p-1 \geq 2 q+r+2$. Then, by $(i i i)_{<L-1}$, the action of $E_{n-p-1}(\mathbf{A})$ on $H_{q}\left(V_{n-p-1}(\mathbf{A})\right)$ is pro trivial. Hence, there exists $s(m) \geq m$ for each $m \in \Xi$ such that the composite 
$\Psi_{m}$

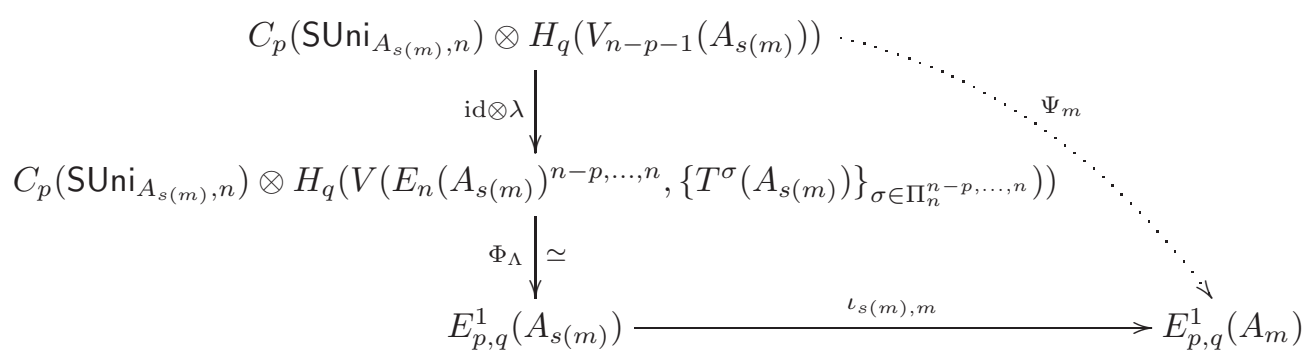

does not depend on the choice of $\Lambda$. We may assume $s(m+1)>s(m)$ for every $m$, so that we obtain a morphism of pro abelian groups

$$
\Psi: C_{p}\left(\mathrm{SUni}_{\mathbf{A}, n}\right) \otimes H_{q}\left(V_{n-p-1}(\mathbf{A})\right) \rightarrow E_{p, q}^{1}(\mathbf{A}) .
$$

Since $\lambda$ is a pro isomorphism and $\Phi_{\Lambda}$ is an isomorphism, we see that $\Psi$ is a pro isomorphism.

Now, by $(i i)_{<L-1}$, the action of $\Sigma_{n-p-1}$ on $H_{q}\left(V_{n-p-1}(\mathbf{A})\right)$ is also pro trivial. Hence, by modifying $s(m) \geq m$ if necessary, we see that the diagram

$$
\begin{aligned}
& C_{p+1}\left(\mathrm{SUni}_{A_{s(m), n}}\right) \otimes H_{q}\left(V_{n-p-2}\left(A_{s(m)}\right)\right) \stackrel{\iota_{s(m), m} \Phi_{\Lambda}(\mathrm{id} \otimes \lambda)}{\longrightarrow} E_{p+1, q}^{1}\left(A_{m}\right)
\end{aligned}
$$

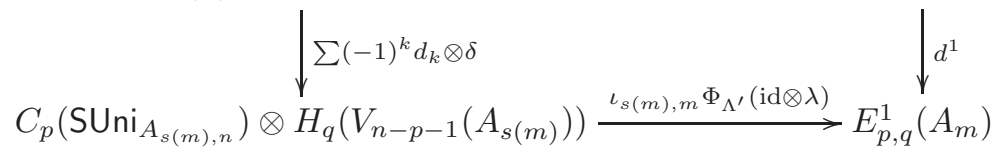

commutes, cf. the formula (4.2). The horizontal maps are the maps $\Psi_{m}$ unless $n-p-1=2 q+r+2$; in the last case only the bottom horizontal map can be identified with $\Psi_{m}$. Consequently, for $q<L-1$, we obtain a morphism of pro complexes

$$
\Psi: \sigma_{\leq n-2 q-r-3}\left(C_{\bullet}\left(\operatorname{SUni}_{\mathbf{A}, n}\right) \otimes H_{q}\left(V_{n-1-\bullet}(\mathbf{A})\right)\right) \rightarrow \sigma_{\leq n-2 q-r-3} E_{\bullet, q}^{1}(\mathbf{A})
$$

and it is a pro isomorphism.

Claim 4.7. For $q<L-1$ and $0<p \leq n-2 q-r-3$,

$$
E_{p, q}^{2}(\mathbf{A})=0 \text {. }
$$

Proof. Suppose that $q<L-1$ and $0<p \leq n-2 q-r-3$. Put $F_{p, q}(\mathbf{A}):=C_{p}\left(\mathrm{SUni}_{\mathbf{A}, n}\right) \otimes$ $H_{q}\left(V_{n-p-1}(\mathbf{A})\right)$, which we regard as a complex in $p$ with the differential $\partial:=\sum(-1)^{k} d_{k} \otimes \delta$. First, we show that $H_{p}\left(F_{\bullet}, q(\mathbf{A})\right)=0$.

By $(i)_{<L-1}$, the canonical map $H_{q}\left(V_{n-p-1}(\mathbf{A})\right) \rightarrow H_{q}\left(V_{n-p}(\mathbf{A})\right)$ is a pro isomorphism, and thus

$$
\operatorname{ker}\left(F_{p, q}(\mathbf{A}) \rightarrow F_{p-1, q}(\mathbf{A})\right) \simeq Z_{p}\left(\operatorname{SUni}_{\mathbf{A}, n}\right) \otimes H_{q}\left(V_{n-p-1}(\mathbf{A})\right),
$$

where $Z_{p}\left(\mathrm{SUni}_{\mathbf{A}, n}\right):=\operatorname{ker}\left(C_{p}\left(\mathrm{SUni}_{\mathbf{A}, n}\right) \rightarrow C_{p-1}\left(\mathrm{SUni}_{\mathbf{A}, n}\right)\right)$. According to Corollary 4.5, the differential

$$
C_{p+1}\left(\text { SUni }_{\mathbf{A}, n}\right) \rightarrow Z_{p}\left(\text { SUni }_{\mathbf{A}, n}\right)
$$

is a pro epimorphism. Also, by $(i)_{<L-1}$, the canonical map

$$
H_{q}\left(V_{n-p-2}(\mathbf{A})\right) \rightarrow H_{q}\left(V_{n-p-1}(\mathbf{A})\right)
$$

is a pro epimorphism. These imply that $\partial: F_{p+1}(\mathbf{A}) \rightarrow \operatorname{ker}\left(F_{p, q}(\mathbf{A}) \rightarrow F_{p-1, q}(\mathbf{A})\right)$ is a pro epimorphism, hence $H_{p}\left(F_{\bullet}, q(\mathbf{A})\right)=0$.

If $p<n-2 L-r-3$, then $\Psi$ (4.3) induces a pro isomorphism

$$
H_{p} F_{\bullet, q}(\mathbf{A}) \simeq E_{p, q}^{2}(\mathbf{A}) .
$$

Hence, in this case, the vanishing of $E_{p, q}^{2}(\mathbf{A})$ follows from the one of $H_{p}\left(F_{\bullet}, q(\mathbf{A})\right)$. 
Finally, let $p=n-2 q-r-3$. Then we have a commutative diagram

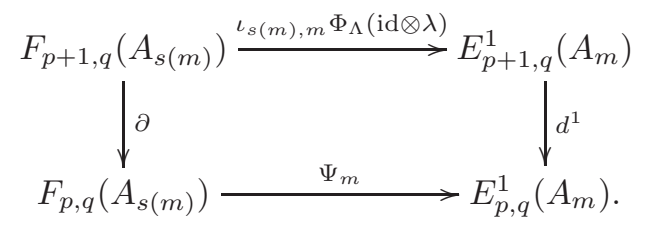

Let $m^{\prime} \geq m$ and $x \in \operatorname{ker}\left(E_{p, q}^{1}\left(A_{m^{\prime}}\right) \rightarrow E_{p-1, q}^{1}\left(A_{m^{\prime}}\right)\right)$. Since $\Psi$ is a pro isomorphism, if we have taken $m^{\prime}$ large enough, $\iota_{m^{\prime}, m} x$ lifts to $y \in \operatorname{ker}\left(F_{p, q}\left(A_{s(m)}\right) \rightarrow F_{p-1, q}\left(A_{s(m)}\right)\right)$ along $\Psi_{m}$. Further, since $H_{p, q}\left(F_{\bullet, q}(\mathbf{A})\right)=0$, we may assume that $y=\partial z$ for some $z \in F_{p+1, q}\left(A_{s(m)}\right)$. Hence, $\iota_{m^{\prime}, m} x$ is in the image of the differential $d^{1}$. This proves $E_{p, q}^{2}(\mathbf{A})=0$.

4.4.3. Conclusion. Suppose that $n \geq 2 L+r$. If $p+q=L-1$ and $p>0$, then $q<L-1$ and $0<p \leq n-2 q-r-3$. Hence, by Claim 4.7, the $E_{p, q}^{2}$-terms with $p+q=L-1$ are zero unless $E_{0, L-1}^{2}$, and the edge map

$$
E_{0, L-1}^{1}(\mathbf{A}) \rightarrow H_{L-1}\left(V_{n}(\mathbf{A})\right)
$$

is a pro epimorphism.

Now, the composite

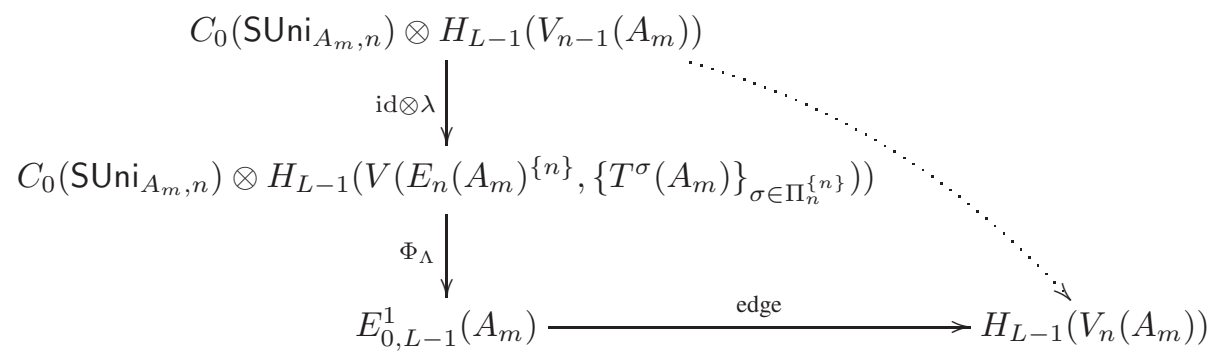

is given by $f \otimes u \mapsto \sigma_{\{i\}}(\delta u) \sigma_{\{i\}}^{-1} \Lambda(f)$, where $f$ is an $A_{m}$-unimodular function with $\operatorname{dom} f=\{i\}$ and $u \in H_{L-1}\left(V_{n-1}\left(A_{m}\right)\right)$. Since the action of $E_{n}(\mathbf{A})$ on the image of $\delta: H_{L-1}\left(V_{n-1}(\mathbf{A})\right) \rightarrow$ $H_{L-1}\left(V_{n}(\mathbf{A})\right)$ is pro trivial by Corollary 4.3, the above composite yields a pro morphism

$$
C_{0}\left(\text { SUni }_{\mathbf{A}, n}\right) \otimes H_{L-1}\left(V_{n-1}(\mathbf{A})\right) \rightarrow H_{L-1}\left(V_{n}(\mathbf{A})\right), \quad f \otimes u \mapsto \sigma_{\{i\}}(\delta u) \sigma_{\{i\}}^{-1} .
$$

Furthermore, since the edge map is a pro epimorphism, $\Phi_{\Lambda}$ is an isomorphism and (id $\otimes \lambda$ ) is a pro isomorphism, we see that (4.4) is a pro epimorphism.

By Corollary 4.3 again, we conclude that the action of $E_{n}(\mathbf{A})$ on $H_{L-1}\left(V_{n}(\mathbf{A})\right)$ is pro trivial. This proves $(\text { iii })_{l=L-1}$.

4.5. Step 2: $V$ to $E$. Suppose that $(i i i)_{l \leq L-1}$ and $(v i)_{l<L+1}$ hold. We show $(v i)_{l=L+1}$.

Suppose that $n \geq 2 L+r$ and fix $k \geq 0$. We set

$$
\tilde{E}_{n}(\mathbf{A}):=\left(\begin{array}{cc}
E_{n}(\mathbf{A}) & * \\
0 & 1_{k}
\end{array}\right), \quad \tilde{T}^{\sigma}(\mathbf{A}):=\left(\begin{array}{cc}
T^{\sigma}(\mathbf{A}) & * \\
0 & 1_{k}
\end{array}\right)
$$

and $\tilde{V}_{n}(\mathbf{A}):=V\left(\tilde{E}_{n}(\mathbf{A}),\left\{\tilde{T}^{\sigma}(\mathbf{A})\right\}_{\sigma \in \Pi_{n}}\right)$.

By Lemma 4.1, the canonical inclusion and projection $V_{n}(\mathbf{A}) \rightleftarrows \tilde{V}_{n}(\mathbf{A})$ are mutually inverse homotopy equivalences. It follows that the action of $\left(\begin{array}{cc}1_{n} & * \\ 0 & 1_{k}\end{array}\right)$ on $H_{*}\left(\tilde{V}_{n}(\mathbf{A})\right)$ is trivial. By $(i i i) \leq L-1$, the action of $E_{n}(\mathbf{A})$ on $H_{q}\left(V_{n}(\mathbf{A})\right) \simeq H_{q}\left(\tilde{V}_{n}(\mathbf{A})\right)$ is pro trivial for $q \leq L-1$. Hence, the action of $\tilde{E}_{n}(\mathbf{A})$ on $H_{q}\left(\tilde{V}_{n}(\mathbf{A})\right)$ is pro trivial for $q \leq L-1$. 
Consider the spectral sequences (4.1) and the canonical map between them;

$$
\begin{gathered}
E_{p, q}^{2}(\mathbf{A})=H_{p}\left(E_{n}(\mathbf{A}), H_{q}\left(V_{n}(\mathbf{A})\right)\right) \Longrightarrow H_{p+q}\left(\bigcup_{\sigma \in \Pi_{n}} B T^{\sigma}(\mathbf{A})\right) \\
\downarrow \\
\tilde{E}_{p, q}^{2}(\mathbf{A})=H_{p}\left(\tilde{E}_{n}(\mathbf{A}), H_{q}\left(\tilde{V}_{n}(\mathbf{A})\right)\right) \Longrightarrow H_{p+q}\left(\bigcup_{\sigma \in \Pi_{n}} B \tilde{T}^{\sigma}(\mathbf{A})\right) .
\end{gathered}
$$

For $q \leq L-1$, the $E^{2}$-terms fit into the extensions

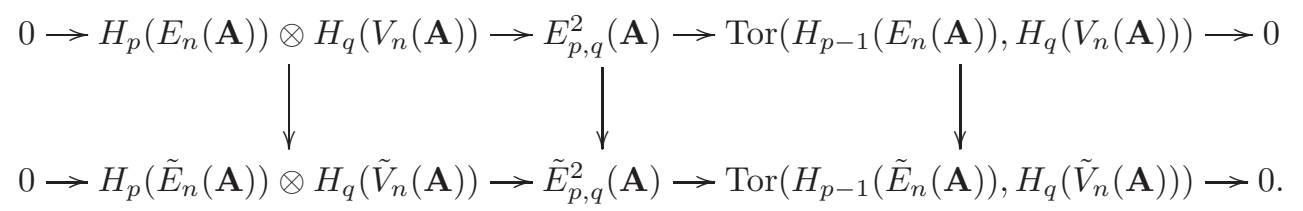

By $(i v)_{<L+1}$, the canonical map $H_{p}\left(E_{n}(\mathbf{A})\right) \rightarrow \tilde{H}_{p}\left(E_{n}(\mathbf{A})\right)$ is a pro isomorphism for $p \leq L$. Hence, the canonical map

$$
E_{p, q}^{2}(\mathbf{A}) \rightarrow \tilde{E}_{p, q}^{2}(\mathbf{A})
$$

is a pro isomorphism for $p \leq L$ and $q \leq L-1$. Also, $E_{0, q}^{2}(\mathbf{A}) \simeq \tilde{E}_{0, q}^{2}(\mathbf{A})$ for all $q \geq 0$, since $H_{*}\left(V_{n}(\mathbf{A})\right) \simeq H_{*}\left(\tilde{V}_{n}(\mathbf{A})\right)$. Finally, by Theorem 3.9, the canonical map $E_{i}^{\infty}(\mathbf{A}) \rightarrow \tilde{E}_{i}^{\infty}(\mathbf{A})$ is a pro isomorphism for $n \geq 2 i$.

Bringing these together, we have:

(1) $E_{p, q}^{2}(\mathbf{A}) \simeq \tilde{E}_{p, q}^{2}(\mathbf{A})$ for $p+q=L-1$.

(2) $E_{p, q}^{2}(\mathbf{A}) \simeq \tilde{E}_{p, q}^{2}(\mathbf{A})$ for $p+q=L$.

(3) $E_{p, q}^{2}(\mathbf{A}) \simeq \tilde{E}_{p, q}^{2}(\mathbf{A})$ for $p+q=L+1$ and $p \geq 2$ and $q \geq 1$.

(4) $E_{L}^{\infty}(\mathbf{A}) \simeq \tilde{E}_{L}^{\infty}(\mathbf{A})$ and $E_{L+1}^{\infty}(\mathbf{A}) \simeq \tilde{E}_{L+1}^{\infty}(\mathbf{A})$.

Then, by Lemma 4.8 below, we conclude that

$$
E_{L+1,0}^{2}(\mathbf{A}) \rightarrow \tilde{E}_{L+1,0}^{2}(\mathbf{A})
$$

is a pro epimorphism, and thus a pro isomorphism. This proves $(v i)_{l=L+1}$.

Lemma 4.8 ([Su96, Remark A.5]). Let $\mathcal{A}$ be an abelian category. Let $f: E \rightarrow \tilde{E}$ be a morphism of first quadrant homological spectral sequence in $\mathcal{A}$, and let $L \geq 0$. Assume that $f$ induces:

(1) A monomorphism $E_{p, q}^{2} \hookrightarrow \tilde{E}_{p, q}^{2}$ for $p+q=L-1$.

(2) An isomorphism $E_{p, q}^{2} \stackrel{\sim}{\rightarrow} \tilde{E}_{p, q}^{2}$ for $p+q=L$.

(3) An epimorphism $E_{p, q}^{2} \rightarrow \tilde{E}_{p, q}^{2}$ for $p+q=L+1, q \geq 1$ and $p \geq 2$.

(4) An isomorphism $E_{L}^{\infty} \stackrel{\sim}{\longrightarrow} \tilde{E}_{L}^{\infty}$ and an epimorphism $E_{L+1}^{\infty} \rightarrow \tilde{E}_{L+1}^{\infty}$.

Then $f$ induces an epimorphism

$$
E_{L+1,0}^{2} \rightarrow \tilde{E}_{L+1,0}^{2} .
$$

4.6. Step 3: Covering argument II. Suppose that $(i v)_{l \leq L+1}$ and $(v, v i)_{l<L+1}$ hold. We show $(v, v i)_{l=L+1}$.

Sublemma 4.9. For $l \leq L+1$ and $n \geq 2 l+r-2$, the conjugate action of $\mathrm{GL}_{n+1}(\mathbb{Z})$ on the image of

$$
H_{l}\left(E_{n}(\mathbf{A})\right) \rightarrow H_{l}\left(E_{n+1}(\mathbf{A})\right)
$$

is pro trivial.

Proof. The case $l=0,1$ is clear. Suppose that $2 \leq l \leq L+1$ and $n \geq 2 l+r-2$.

Since $\mathrm{GL}_{n+1}(\mathbb{Z})=\mathbb{Z} \times \mathrm{SL}_{n+1}(\mathbb{Z})$ and $\mathrm{SL}_{n+1}(\mathbb{Z})=E_{n+1}(\mathbb{Z}), \mathrm{GL}_{n+1}(\mathbb{Z})$ is generated by $e_{i, n+1}(1)$, $e_{n+1, i}(1), 1 \leq i \leq n$, and $\operatorname{diag}(1, \ldots, 1,-1)$. It is obvious that $\operatorname{diag}(1, \ldots, 1,-1)$ acts trivially on the image of $H_{l}\left(E_{n}(\mathbf{A})\right) \rightarrow H_{l}\left(E_{n+1}(\mathbf{A})\right)$.

We show the triviality of the conjugate action of $e_{i, n+1}(1)$; the one of $e_{n+1, i}(1)$ is similar. By Corollary 1.3 , it suffices to show that the action on the image of

$$
H_{l}\left(E_{n}(\mathbf{R}, \mathbf{A})\right) \rightarrow H_{l}\left(E_{n+1}(\mathbf{R}, \mathbf{A})\right)
$$


is pro trivial for some unital pro ring $\mathbf{R}$ which contains $\mathbf{A}$ as a two-sided ideal. The inclusion $E_{n}(\mathbf{R}, \mathbf{A}) \hookrightarrow$ $E_{n+1}(\mathbf{R}, \mathbf{A})$ factors through

$$
\tilde{E}_{n}(\mathbf{R}, \mathbf{A}):=\left(\begin{array}{cc}
E_{n}(\mathbf{R}, \mathbf{A}) & * \\
0 & 1
\end{array}\right) \subset E_{n+1}(\mathbf{R}, \mathbf{A})
$$

and it is normalized by $e_{i, n+1}(1)$. Hence, it suffices to show that $e_{i, n+1}(1)$ acts pro trivially on the image of $H_{l}\left(E_{n}(\mathbf{R}, \mathbf{A})\right) \rightarrow H_{l}\left(\tilde{E}_{n}(\mathbf{R}, \mathbf{A})\right)$. Now, we have a commutative diagram

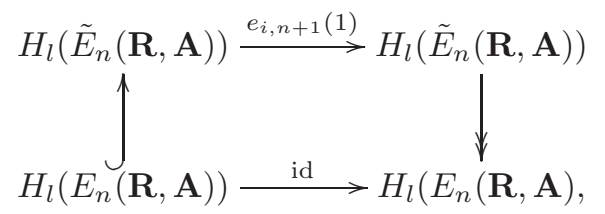

and the vertical maps, the canonical inclusion and projection, are pro isomorphisms by $(i v)_{\leq L+1}$. This implies that $e_{i, n+1}(1)$ acts pro trivially on the image of $H_{l}\left(E_{n}(\mathbf{R}, \mathbf{A})\right) \rightarrow H_{l}\left(\tilde{E}_{n}(\mathbf{R}, \mathbf{A})\right)$.

We consider the hyperhomology spectral sequence

$$
E_{p, q}^{1}(\mathbf{A})=H_{q}\left(E_{n+1}(A), C_{p}\left(\operatorname{SUni}_{\mathbf{A}, n+1}\right)\right) \Rightarrow H_{p+q}\left(E_{n+1}(\mathbf{A}), C \bullet\left(\operatorname{SUni}_{\mathbf{A}, n+1}\right)\right) .
$$

The $C_{p}\left(\mathrm{SUni}_{\mathbf{A}, n+1}\right)$ decomposes into a direct sum of $E_{n+1}(A)$-submodules $C_{p}\left(\mathrm{SUni}_{\mathbf{A}, n+1}^{I}\right)$ with $|I|=$ $p+1$, and we have a levelwise isomorphism $\mathbb{Z} E_{n+1}(\mathbf{A}) \otimes_{\mathbb{Z} E_{n+1}(\mathbf{A})^{I}} \mathbb{Z} \stackrel{\sim}{\rightarrow} C_{p}\left(\mathrm{SUni}_{\mathbf{A}, n+1}\right)^{I}$, which sends $\alpha \in E_{n+1}(\mathbf{A})$ to the unimodular function $i \mapsto e_{i} \alpha, i \in I$. Hence,

$$
\bigsqcup_{|I|=p+1} H_{q}\left(E_{n+1}(\mathbf{A})^{I}\right) \simeq E_{p, q}^{1}(\mathbf{A}) .
$$

Let $\Delta^{n}$ be the nerve of the partially ordered set $\{1<2<\cdots<n+1\}$. We define level maps $E_{n-p}(\mathbf{A}) \rightarrow E_{n+1}(\mathbf{A})^{I}$ by sending $\alpha$ to $\sigma_{I}\left(\begin{array}{cc}\alpha & 0 \\ 0 & 1_{p+1}\end{array}\right) \sigma_{I}^{-1}$, where $\sigma_{I}$ is the shuffle permutation $\sigma_{I}\{n-$ $p+1, \ldots, n+1\}=I$. These maps yield

$$
\Psi: \Delta_{p}^{n} \otimes H_{q}\left(E_{n-p}(\mathbf{A})\right) \simeq \bigsqcup_{|I|=p+1} H_{q}\left(E_{n-p}(\mathbf{A})\right) \rightarrow \bigsqcup_{|I|=p+1} H_{q}\left(E_{n+1}(\mathbf{A})^{I}\right) \simeq E_{p, q}^{1}(\mathbf{A}) .
$$

It follows from Theorem 1.6 and $(i v)_{\leq L+1}$ that $\Psi$ is a pro isomorphism for $q \leq L+1$ and $n-p \geq$ $\max (2 q+r-2, r+1)$. Furthermore, by Sublemma 4.9, we see that the diagram

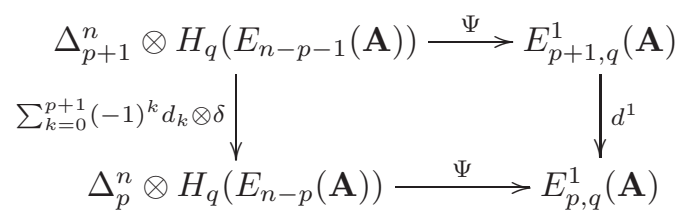

commutes for $q \leq L+1$ and $n-p \geq 2 q+r-1$, where $d_{k}$ are the face maps of $\Delta^{n}$ and $\delta$ is the canonical $\operatorname{map} H_{q}\left(E_{n-p-1}(\mathbf{A})\right) \rightarrow H_{q}\left(E_{n-p}(\mathbf{A})\right)$.

Claim 4.10. For $q \leq L$ and $0<p \leq n-2 q-r+1$,

$$
E_{p, q}^{2}(\mathbf{A})=0 \text {. }
$$

Proof. Suppose that $q \leq L$ and $0<p \leq n-2 q-r+1$. Put $F_{p, q}(\mathbf{A}):=\Delta_{p}^{n} \otimes H_{q}\left(E_{n-p}(\mathbf{A})\right)$, which we regard as a complex in $p$ with differential $\sum_{k=0}^{p+1}(-1)^{k} d_{k} \otimes \delta$. Then, by $(v i)_{<L+1}$, we have

$$
\operatorname{ker}\left(F_{p, q}(\mathbf{A}) \rightarrow F_{p-1, q}(\mathbf{A})\right) \simeq \operatorname{ker}\left(\mathbb{Z} \Delta_{p}^{n} \rightarrow \mathbb{Z} \Delta_{p-1}^{n}\right) \otimes H_{q}\left(E_{n-p}(\mathbf{A})\right) .
$$

Again by $(v i)_{<L+1}$, the canonical map

$$
H_{q}\left(E_{n-p-1}(\mathbf{A})\right) \rightarrow H_{q}\left(E_{n-p}(\mathbf{A})\right)
$$

is a pro epimorphism. Since $\Delta^{n}$ is contractible, we conclude that $H_{p}\left(F_{\bullet}, q(\mathbf{A})\right)=0$. 
Now, we have a pro isomorphism

$$
E_{p, q}^{2}(\mathbf{A}) \simeq H_{p}\left(F_{\bullet, q}(\mathbf{A})\right)
$$

for $n-p-1 \geq r+1$. Our assumption says $n-p-1 \geq 2 q+r-2$; hence, in case $2 q+r-2 \geq r+1$, the vanishing of $E_{p, q}^{2}(\mathbf{A})$ follows form the one of $H_{p}\left(F_{\bullet}, q(\mathbf{A})\right)$.

It remains to show the case $q=1$. However, in this case,

$$
E_{p, 1}^{1}(\mathbf{A}) \underset{\Psi}{\stackrel{\sim}{\longrightarrow}} \Delta^{n} \otimes H_{1}\left(E_{n-p}(\mathbf{A})\right)=0 .
$$

This completes the proof.

Suppose that $n \geq 2 L+r$. Then the $E^{2}$-terms with $p+q=L+1$ are zero unless $E_{0, L+1}^{2}(\mathbf{A})$. Hence, the edge map

$$
E_{0, L+1}^{1}(\mathbf{A}) \rightarrow E_{L+1}^{\infty}(\mathbf{A})
$$

is a pro epimorphism. The left hand side is pro isomorphic to $\Delta_{0}^{n} \otimes H_{L+1}\left(E_{n}(\mathbf{A})\right)$ by $\Psi$. According to Corollary 4.5, $\tilde{H}_{i}\left(C_{*}\left(\mathrm{SUni}_{\mathbf{A}, n+1}\right)\right)=0$ for $n \geq i+r$. Hence, we have a pro isomorphism

$$
E_{L+1}^{\infty}(\mathbf{A})=H_{L+1}\left(E_{n+1}(\mathbf{A}), C_{\bullet}\left(\operatorname{SUni}_{\mathbf{A}, n+1}\right)\right) \simeq H_{L+1}\left(E_{n+1}(\mathbf{A})\right) .
$$

By using Sublemma 4.9, we see that the edge map

$$
\Delta_{0}^{n} \otimes H_{L+1}\left(E_{n}(\mathbf{A})\right) \rightarrow H_{L+1}\left(E_{n+1}(\mathbf{A})\right)
$$

coincides as a pro morphism with the sum of the canonical map $\delta: H_{L+1}\left(E_{n}(\mathbf{A})\right) \rightarrow H_{L+1}\left(E_{n+1}(\mathbf{A})\right)$. Hence, the $\delta$ is a pro epimorphism. This proves the first half of $(v i)_{l=L+1}$.

Next, suppose that $n \geq 2 L+r+1$. Then by Claim 4.10, $E_{s, L-s+2}^{s}(\mathbf{A})=0$ for $s \geq 2$. Hence, we have an exact sequence

$$
\Delta_{1}^{n} \otimes H_{L+1}\left(E_{n-1}(\mathbf{A})\right) \longrightarrow \Delta_{0}^{n} \otimes H_{L+1}\left(E_{n}(\mathbf{A})\right) \longrightarrow H_{L+1}\left(E_{n+1}(\mathbf{A})\right) \longrightarrow 0 .
$$

Since $H_{L+1}\left(E_{n-1}(\mathbf{A})\right) \rightarrow H_{L+1}\left(E_{n}(\mathbf{A})\right)$ is a pro epimorphism, we conclude that the canonical map

$$
H_{L+1}\left(E_{n}(\mathbf{A})\right) \stackrel{\sim}{\rightarrow} H_{L+1}\left(E_{n+1}(\mathbf{A})\right)
$$

is a pro isomorphism. This proves the second half of $(v i)_{l=L+1}$.

Finally, since $H_{L+1}\left(E_{n-1}(\mathbf{A})\right) \rightarrow H_{L+1}\left(E_{n}(\mathbf{A})\right)$ is a pro epimorphism, Sublemma 4.9 implies that the action of $\Sigma_{n}$ on $H_{L+1}\left(E_{n}(\mathbf{A})\right)$ is pro trivial. This proves $(v)_{l=L+1}$

4.7. Step 4: $E$ to $V$. Suppose that $(i, i i)_{l<L-1},(i i i)_{l \leq L-1}$ and $(v i)_{l \leq L+1}$ hold. We show $(i, i i)_{l \leq L-1}$.

Suppose that $n \geq 2 L+r$. Consider the spectral sequences (4.1) and the canonical morphism between them;

$$
\begin{gathered}
{ }^{n} E_{p, q}^{2}(\mathbf{A})=H_{p}\left(E_{n}(\mathbf{A}), H_{q}\left(V_{n}(\mathbf{A})\right)\right) \Longrightarrow H_{p+q}\left(\bigcup_{\sigma \in \Pi_{n}} B T^{\sigma}(\mathbf{A})\right) \\
\downarrow \\
{ }^{n+1} E_{p, q}^{2}(\mathbf{A})=H_{p}\left(E_{n+1}(\mathbf{A}), H_{q}\left(V_{n+1}(\mathbf{A})\right)\right) \Longrightarrow H_{p+q}\left(\bigcup_{\sigma \in \Pi_{n+1}} B T^{\sigma}(\mathbf{A})\right) .
\end{gathered}
$$

By $(i i i)_{\leq L-1}$, for $q \leq L-1$, the $E^{2}$-terms fit into the extensions

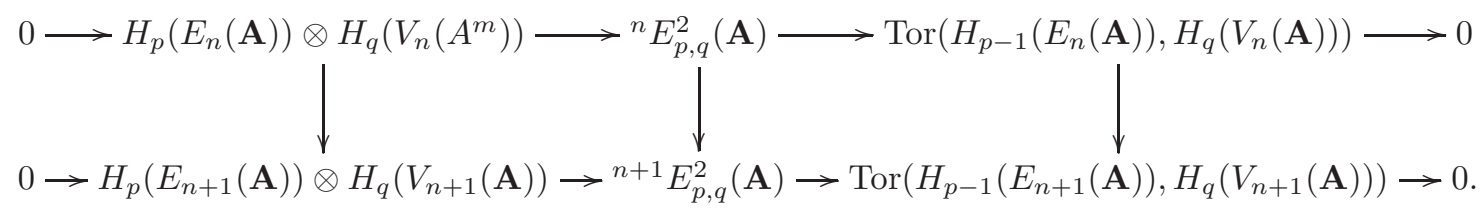

Hence, it follows from $(i)_{<L-1}$ and $(v i)_{\leq L+1}$ that the map

$$
{ }^{n} E_{p, q}^{2}(\mathbf{A}) \rightarrow{ }^{n+1} E_{p, q}^{2}(\mathbf{A})
$$

is a pro epimorphism for $q<L-1$ and $p \leq L+1$, and it is a pro isomorphism if further $n \geq 2 p+r-1$.

Finally, by Theorem 3.9, ${ }^{n} E_{i}^{\infty}(\mathbf{A}) \simeq{ }^{n+1} E_{i}^{\infty}(\mathbf{A})$ for $n \geq 2 i+1$.

Bringing these together, we have:

(1) ${ }^{n} E_{p, q}^{2}(\mathbf{A}) \simeq{ }^{n+1} E_{p, q}^{2}(\mathbf{A})$ for $p+q=L-1$ and $p \geq 1$. 
(2) ${ }^{n} E_{p, q}^{2}(\mathbf{A}) \simeq{ }^{n+1} E_{p, q}^{2}(\mathbf{A})$ for $p+q=L$ and $p \geq 2$.

(3) ${ }^{n} E_{p, q}^{2}(\mathbf{A}) \rightarrow{ }^{n+1} E_{p, q}^{2}(\mathbf{A})$ for $p+q=L+1$ and $p \geq 3$.

(4) ${ }^{n} E_{L-1}^{\infty}(\mathbf{A}) \simeq{ }^{n+1} E_{L-1}^{\infty}(\mathbf{A})$ and ${ }^{n} E_{L}^{\infty}(\mathbf{A}) \simeq{ }^{n+1} E_{L}^{\infty}(\mathbf{A})$.

Then, by Lemma 4.11 below, we conclude that the canonical map

$$
{ }^{n} E_{0, L-1}^{2}(\mathbf{A}) \stackrel{\sim}{\rightarrow}{ }^{n+1} E_{0, L-1}^{2}(\mathbf{A})
$$

is a pro isomorphism. By $(i i i)_{\leq L-1}$, the left hand side (resp. right hand side) is pro isomorphic to $H_{L-1}\left(V_{n}(\mathbf{A})\right)\left(\right.$ resp. $\left.H_{L-1}\left(V_{n+1}(\mathbf{A})\right)\right)$. Hence, we get the second part of $(i)_{l=L-1}$.

Next, we show $(\text { ii })_{l=L-1}$. Now, the canonical map

$$
H_{L-1}\left(V_{n}(\mathbf{A})\right) \stackrel{\sim}{\rightarrow} H_{L-1}\left(V_{n+2}(\mathbf{A})\right)
$$

is a $\Sigma_{n}$-equivariant pro isomorphism. Hence, it suffices to show that $\Sigma_{n+2}$ (and thus $\Sigma_{n}$ ) acts pro trivially on $H_{L-1}\left(V_{n+2}(\mathbf{A})\right)$. Now, the permutation $\tau_{n+1, n+2}$ acts pro trivially on $H_{L-1}\left(V_{n+2}(\mathbf{A})\right)$, since it acts trivially on the image of the above map. Since $\Sigma_{n+2}$ is the normal closure of $\tau_{n+1, n+2}, \Sigma_{n+2}$ also acts pro trivially on $H_{L-1}\left(V_{n+2}(\mathbf{A})\right)$.

In Step 1, we have seen that the map (4.4)

$$
C_{0}\left(\mathrm{SUni}_{\mathbf{A}, n}\right) \otimes H_{L-1}\left(V_{n-1}(\mathbf{A})\right) \rightarrow H_{L-1}\left(V_{n}(\mathbf{A})\right)
$$

sending $f \otimes u \mapsto \sigma_{\{i\}}(\delta u) \sigma_{\{i\}}^{-1}(\operatorname{dom} f=\{i\})$ is a pro epimorphism for $n \geq 2 L+r$. Now, we know that $\sigma_{\{i\}}(\delta u) \sigma_{\{i\}}^{-1}=\delta u$. Therefore, the canonical map $\delta: H_{L-1}\left(V_{n-1}(\mathbf{A})\right) \rightarrow H_{L-1}\left(V_{n}(\mathbf{A})\right)$ is a pro epimorphism. This completes the proof of $(i)_{l=L-1}$.

Lemma 4.11 ([Su96, Theorem A.6]). Let $\mathcal{A}$ be an abelian category. Let $f: E \rightarrow \tilde{E}$ be a morphism of first quadrant homological spectral sequence in $\mathcal{A}$, and let $L>0$. Assume that $f$ induces:

(1) A monomorphism $E_{p, q}^{2} \hookrightarrow \tilde{E}_{p, q}^{2}$ for $p+q=L-1, p \geq 1$.

(2) An isomorphism $E_{p, q}^{2} \stackrel{\sim}{\longrightarrow} \tilde{E}_{p, q}^{2}$ for $p+q=L, p \geq 2$.

(3) An epimorphism $E_{p, q}^{2} \rightarrow \tilde{E}_{p, q}^{2}$ for $p+q=L+1, p \geq 3$.

(4) Isomorphisms $E_{L-1}^{\infty} \stackrel{\sim}{\rightarrow} \tilde{E}_{L-1}^{\infty}$ and $E_{L}^{\infty} \stackrel{\sim}{\rightarrow} \tilde{E}_{L}^{\infty}$.

Then $f$ induces an isomorphism

$$
E_{0, L-1}^{2} \stackrel{\simeq}{\rightarrow} \tilde{E}_{0, L-1}^{2}
$$

4.8. Homology pro stability for $\mathrm{GL}_{n}$. Now, we can prove Theorem 0.3 . We restate it here.

Theorem 4.12. Let $\mathbf{A}$ be a commutative Tor-unital pro ring. Let $r=\max (\operatorname{sr}(\mathbf{A}), 2)$ and $l \geq 0$. Then the canonical map

$$
H_{l}\left(\mathrm{GL}_{n}(\mathbf{A})\right) \rightarrow H_{l}\left(\mathrm{GL}_{n+1}(\mathbf{A})\right)
$$

is a pro epimorphism for $n \geq 2 l+r-2$ and a pro isomorphism for $n \geq 2 l+r-1$.

Proof. The case $l=0$ is clear. The case $l=1$ is proved in Theorem 1.5. Let $l \geq 2$ and $n \geq 2 l+r-2$. Then, by Theorem 1.5 and Corollary 1.3, the sequence

$$
0 \longrightarrow E_{n}(\mathbf{A}) \longrightarrow \mathrm{GL}_{n}(\mathbf{A}) \longrightarrow H_{1}(\mathrm{GL}(\mathbf{A})) \longrightarrow 0
$$

is exact up to pro isomorphisms. Now, we have a morphism of spectral sequences;

$$
\begin{gathered}
{ }^{n} E_{p, q}^{2}=H_{p}\left(H_{1}(\mathrm{GL}(\mathbf{A})), H_{q}\left(E_{n}(\mathbf{A})\right)\right) \\
\downarrow \\
{ }^{n+1} E_{p, q}^{2}=H_{p}\left(H_{1}(\mathrm{GL}(\mathbf{A})), H_{q}\left(E_{n+1}(\mathbf{A})\right)\right) \Longrightarrow H_{p+q}\left(\mathrm{GL}_{n}(\mathbf{A})\right) \\
\downarrow \\
\downarrow
\end{gathered}
$$

Using these spectral sequences, we can easily deduce the theorem from Theorem 4.6 (vi).

Corollary 4.13. Let $\mathbf{B}$ be a pro ring with a two-sided ideal $\mathbf{A}$ and $r=\max (\operatorname{sr}(\mathbf{A}), 2)$. Suppose that $\mathbf{A}$ is commutative and Tor-unital. Then the conjugate action of $\mathrm{GL}_{n}(\mathbf{B})$ on $H_{l}\left(\mathrm{GL}_{n}(\mathbf{A})\right)$ is pro trivial for $n \geq 2 l+r-1$. 
Proof. Let $\alpha$ (resp. $\beta$ ) be the map $\mathrm{GL}_{n} \rightarrow \mathrm{GL}_{2 n}$ given by

$$
g \mapsto\left(\begin{array}{cc}
g & 0 \\
0 & 1_{n}
\end{array}\right) \quad \text { resp. } g \mapsto\left(\begin{array}{cc}
1_{n} & 0 \\
0 & g
\end{array}\right) .
$$

According to the Theorem 4.12, the induced maps

$$
\alpha, \beta: H_{l}\left(\mathrm{GL}_{n}(\mathbf{A})\right) \stackrel{\sim}{\longrightarrow} H_{l}\left(\mathrm{GL}_{2 n}(\mathbf{A})\right)
$$

are pro isomorphisms for $n \geq 2 l+r-1$.

Write $\mathbf{B}=\left\{B_{m}\right\}_{m \in J}$ and $\mathbf{A}=\left\{A_{m}\right\}_{m \in J}$. For each $m \in J$, choose $s(m) \geq m$ such that if $\alpha(a)=0$ with $a \in H_{l}\left(\mathrm{GL}_{n}\left(A_{s(m)}\right)\right)$ then $\iota_{s(m), m}(a)=0$. Next, choose $t(m) \geq s(m)$ such that for every $x \in H_{l}\left(\mathrm{GL}_{n}\left(A_{t(m)}\right)\right)$ there exists $y \in H_{l}\left(\mathrm{GL}_{n}\left(A_{s(m)}\right)\right)$ with $\iota_{t(m), s(m)}(\alpha(x))=\beta(y)$. Then, for $g \in \mathrm{GL}_{n}\left(B_{t(m)}\right)$ and $x \in H_{l}\left(\mathrm{GL}_{n}\left(A_{t(m)}\right)\right)$,

$$
\begin{aligned}
\alpha\left(\iota_{t(m), s(m)}(g x)\right) & =\alpha\left(\iota_{t(m), s(m)}(g)\right) \beta(y) \\
& =\beta(y) \\
& =\alpha\left(\iota_{t(m), s(m)}(x)\right) .
\end{aligned}
$$

Hence, $\iota_{t(m), m}(g x)=\iota_{t(m), m}(x)$. This completes the proof.

Suslin has shown that if a ring $A$ is Tor-unital then for every ring $B$ which contains $A$ as a two-sided ideal the conjugate action of $\mathrm{GL}(B)$ on $H_{l}(\mathrm{GL}(A))$ is trivial, cf. [Su95, Corollary 4.5], see also [SW92, Corollary 1.6]. Geisser-Hesselholt has generalized Suslin's result to a pro setting, cf. [GH06, Proposition 1.3]. Here is a straightforward generalization of their result.

Theorem 4.14 (Suslin, Geisser-Hesselholt). Let $\mathbf{B}$ be a pro ring with a two-sided ideal A. Suppose that A is Tor-unital. Then the conjugate action of $\mathrm{GL}(\mathbf{B})$ on $H_{l}(\mathrm{GL}(\mathbf{A}))$ is pro trivial for all $l \geq 0$.

By using Theorem 4.14, we can strengthen our main theorem, Theorem 4.12.

Theorem 4.15. Let $\mathbf{A}$ be a commutative Tor-unital pro ring, $r=\max (\operatorname{sr}(\mathbf{A}), 2)$ and $l \geq 0$. Suppose that there exists a unital pro ring $\mathbf{R}$ with $\operatorname{sr}(\mathbf{R})<\infty$ which contains $\mathbf{A}$ as a two-sided ideal. Then the canonical map

$$
H_{l}\left(\mathrm{GL}_{n}(\mathbf{A})\right) \rightarrow H_{l}(\mathrm{GL}(\mathbf{A}))
$$

is a pro epimorphism for $n \geq 2 l+r-2$ and a pro isomorphism for $n \geq 2 l+r-1$.

Proof. Let $\mathbf{R}$ be a unital pro ring as in the statement. Consider the commutative diagram

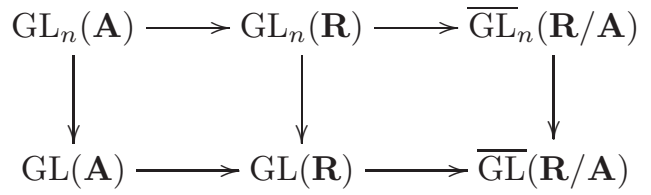

with exact rows. Now, the second and the third maps induce isomorphisms on homology for $n$ large enough. Also, the action of $\mathrm{GL}_{n}(\mathbf{R})$ on $H_{l}\left(\mathrm{GL}_{n}(\mathbf{A})\right)$ is pro trivial for $n$ large enough (Theorem 4.13) and for $n=\infty$ (Theorem 4.14). Consequently, the canonical map

$$
H_{l}\left(\mathrm{GL}_{n}(\mathbf{A})\right) \stackrel{\sim}{\longrightarrow} H_{l}(\mathrm{GL}(\mathbf{A}))
$$

is a pro isomorphism for $n$ large enough. Combining it with Theorem 4.12, we get the result. 


\section{REFERENCES}

[GH06] T. Geisser, L. Hesselholt, Bi-relative algebraic K-theory and topological cyclic homology, Invent. Math. 166, no. 2 (2006). [Is01] D. C. Isaksen, A model structure on the category of pro-simplicial sets, Trans. Amer. Math. Soc. 353, no. 7 (2001).

[IK17] R. Iwasa, W. Kai, Chern classes with modulus, arXiv:1611.07882 (2017).

[Mo15] M. Morrow, Pro unitality and pro excision in algebraic K-theory and cyclic homology, J. reine angew. Math., Ahead of Print (2015).

[Su82] A. A. Suslin, Stability in algebraic K-theory, Lecture Notes in Math., 996, Springer, Berlin (1982).

[Su95] A. A. Suslin, Excision in integer algebraic K-theory, Trudy Mat. Inst. Steklov. 208 (1995).

[Su96] A. A. Suslin, Holomogy stability for H-unital $\mathbb{Q}$-algebras, Amer. Math. Soc. Transl. Ser. 2, 174 (1996).

[SW92] A. A. Suslin, M. Wodzicki, Excision in algebraic K-theory, Ann. of Math. (2) 136 no. 1 (1992).

[Ti76] J. Tits, Systèmes générateurs de groupes de congruence C. R. Acad. Sci. Paris Sér. A 283, no. 9 (1976).

[vdK80] W. van der Kallen, Homology stability for linear groups, Invent. Math. 60, no. 3 (1980).

[Va69] L. N. Vaseršteĭn, On the stabilization of the general linear groups over a ring, Math. USSR-Sb. 8 (1969).

Graduate School of Mathematical Sciences, the University of Tokyo, 3-8-1 Komaba, Meguro-ku, Tokyo, 153-8914 JAPAN.

E-mail address: ryomei@ms.u-tokyo.ac.jp 